\title{
THE 31 DEG $^{2}$ RELEASE OF THE STRIPE 82 X-RAY SURVEY: THE POINT SOURCE CATALOG
}

\author{
Stephanie M. LaMassa ${ }^{1,2,3}$, C. Megan Urry ${ }^{1,2}$, Nico Cappelluti ${ }^{4}$, Hans Böhringer ${ }^{5}$, Andrea Comastri ${ }^{4}$, Eilat Glikman ${ }^{6}$, \\ Gordon Richards $^{7}$, Tonima Ananna ${ }^{1,2}$, Marcella Brusa ${ }^{4,8}$, Carie Cardamone ${ }^{9}$, Gayoung Chon ${ }^{5}$, Francesca Civano ${ }^{1,10}$, \\ Duncan Farrah $^{11}$, Marat Gilfanov ${ }^{12,13}$, Paul Green ${ }^{10}$, S. Komossa ${ }^{14}$, Paulina Lira ${ }^{15}$, Martin Makler ${ }^{16}$, \\ Stefano Marchesi ${ }^{1,4,10}$, Robert Pecoraro ${ }^{1,2}$, Piero Ranalli ${ }^{4,17}$, Mara Salvato ${ }^{5}$, Kevin Schawinski ${ }^{18}$, Daniel Stern ${ }^{19}$, \\ Ezequiel Treister $^{20}$, ANd Marco Viero ${ }^{21,22}$ \\ ${ }^{1}$ Yale Center for Astronomy \& Astrophysics, Physics Department, P.O. Box 208120, New Haven, CT 06520, USA \\ ${ }^{2}$ Department of Physics, Yale University, P.O. Box 208121, New Haven, CT 06520, USA \\ ${ }^{3}$ Now at NASA Goddard Space Flight Center, Greenbelt, MD 20771, USA \\ ${ }_{5}^{4}$ INAF-Osservatorio Astronomico di Bologna, via Ranzani 1, I-40127 Bologna, Italy \\ ${ }^{5}$ Max-Planck-Institut für extraterrestrische Physik, D-85748 Garching, Germany \\ ${ }^{6}$ Department of Physics, Middlebury College, Middlebury, VT 05753, USA \\ ${ }^{7}$ Department of Physics, Drexel University, 3141 Chestnut Street, Philadelphia, PA 19104, USA \\ ${ }^{8}$ DIFADipartimento di Fisica e Astronomia, Universita' di Bologna, viale Berti Pichat 6/2, I-40127 Bologna, Italy \\ 9 Department of Math \& Science, Wheelock College, 200 Riverway, Boston, MA 02215, USA
${ }_{10}$ Smithsonian Astrophysical Observatory, 60 Garden Street, Cambridge, MA 02138, USA \\ ${ }^{11}$ Department of Physics MC 0435, Virginia Polytechnic Institute and State University, 850 West Campus Drive, Blacksburg, VA 24061, USA \\ ${ }_{12}$ Max-Planck Institut für Astrophysik, Karl-Schwarzschild-Str. 1, Postfach 1317, D-85741 Garching, Germany \\ ${ }^{13}$ Space Research Institute of Russian Academy of Sciences, Profsoyuznaya 84/32, 117997 Moscow, Russia \\ ${ }^{14}$ Max-Planck-Institut für Radioastronomie, Auf dem Hügel 69, D-53121 Bonn, Germany \\ ${ }^{15}$ Departamento de Astronomia, Universidad de Chile, Camino del Observatorio 1515, Santiago, Chile \\ ${ }^{16}$ Centro Brasileiro de Pesquisas Físicas, Rua Dr Xavier Sigaud 150, Rio de Janeiro, RJ 22290-180, Brazil \\ ${ }^{17}$ Institute for Astronomy, Astrophysics, Space Applications and Remote Sensing (IAASARS), National Observatory of Athens, 15236 Penteli, Greece \\ ${ }^{18}$ Institute for Astronomy, Department of Physics, ETH Zurich, Wolfgang-Pauli Strasse 27, CH-8093 Zurich, Switzerland \\ 19 Jet Propulsion Laboratory, California Institute of Technology, Pasadena, CA 91109, USA \\ ${ }^{21}$ Kavli Institute for Particle Astrophysics and Cosmology, Stanford University, 382 Via Pueblo Mall, Stanford, CA 94305, USA \\ ${ }^{22}$ California Institute of Technology, 1200 E. California Boulevard, Pasadena, CA 91125, USA \\ Received 2015 September 29; accepted 2015 November 28; published 2016 February 1
}

\begin{abstract}
We release the next installment of the Stripe 82 X-ray survey point-source catalog, which currently covers $31.3 \mathrm{deg}^{2}$ of the Sloan Digital Sky Survey (SDSS) Stripe 82 Legacy field. In total, 6181 unique X-ray sources are significantly detected with XMM-Newton $(>5 \sigma)$ and Chandra $(>4.5 \sigma)$. This catalog release includes data from XMM-Newton cycle AO 13, which approximately doubled the Stripe $82 \mathrm{X}$ survey area. The flux limits of the Stripe $82 \mathrm{X}$ survey are $8.7 \times 10^{-16} \mathrm{erg} \mathrm{s}^{-1} \mathrm{~cm}^{-2}, 4.7 \times 10^{-15} \mathrm{erg} \mathrm{s}^{-1} \mathrm{~cm}^{-2}$, and $2.1 \times 10^{-15} \mathrm{erg} \mathrm{s}^{-1} \mathrm{~cm}^{-2}$ in the soft $(0.5-2 \mathrm{keV})$, hard $(2-10 \mathrm{keV})$, and full bands $(0.5-10 \mathrm{keV})$, respectively, with approximate half-area survey flux limits of $5.4 \times 10^{-15} \mathrm{erg} \mathrm{s}^{-1} \mathrm{~cm}^{-2}, 2.9 \times 10^{-14} \mathrm{erg} \mathrm{s}^{-1} \mathrm{~cm}^{-2}$, and $1.7 \times 10^{-14} \mathrm{erg} \mathrm{s}^{-1} \mathrm{~cm}^{-2}$. We matched the $\mathrm{X}$-ray source lists to available multi-wavelength catalogs, including updated matches to the previous release of the Stripe $82 \mathrm{X}$ survey; $88 \%$ of the sample is matched to a multi-wavelength counterpart. Due to the wide area of Stripe $82 \mathrm{X}$ and rich ancillary multi-wavelength data, including coadded SDSS photometry, mid-infrared WISE coverage, near-infrared coverage from UKIDSS and VISTA Hemisphere Survey, ultraviolet coverage from GALEX, radio coverage from FIRST, and far-infrared coverage from Herschel, as well as existing 30\% optical spectroscopic completeness, we are beginning to uncover rare objects, such as obscured high-luminosity active galactic nuclei at high-redshift. The Stripe $82 \mathrm{X}$ point source catalog is a valuable data set for constraining how this population grows and evolves, as well as for studying how they interact with the galaxies in which they live.
\end{abstract}

Key words: catalogs - galaxies: active - quasars: general - surveys - X-rays: general

Supporting material: FITS files

\section{INTRODUCTION}

Active galactic nuclei (AGNs) signal the growth of supermassive black holes at galactic centers. Studying AGNs over a range of redshift allows us to discover how supermassive black holes evolve over cosmic time to the present day. As AGNs emit energy over a range of wavelengths, they can be identified by various signatures, including optical and ultraviolet light from the accretion disk feeding the black hole (Koratkar \& Blaes 1999), optical emission from gas ionized by accretion disk photons (Baldwin et al. 1981; Veilleux \& Osterbrock 1987; Vanden Berk et al. 2001), X-ray emission from the AGN corona (Haardt \& Maraschi 1991; Brandt \& Alexander 2015), mid-infrared emission from AGN heated circumnuclear dust (Spinoglio \& Malkan 1989; Lacy et al. 2004; Stern et al. 2005; Donley et al. 2012; Stern et al. 2012; Assef et al. 2013), and fine-structure emission lines (Farrah et al. 2007; Weaver et al. 2010; Meléndez et al. 2014), and radio emission from jets launched by the accretion disk (Kellermann et al. 1989; Hooper et al. 1995). These different selection criteria favor different parts of the AGN population, and by combining these methods, a comprehensive view of black hole growth is revealed.

Multi-wavelength surveys are then the key for unlocking the secrets of AGN evolution and how they relate to the galaxies they inhabit. Complementary survey strategies select different 
populations in the redshift-luminosity plane. Deep, pencilbeam surveys uncover the faintest objects in the universe while wide-area surveys are required to discover a representative sampling of rare objects that have a low space density. Such rare sources include high-luminosity AGNs at high-redshift (e.g., $L_{x}>10^{45} \mathrm{erg} \mathrm{s}^{-1}$ at $z>2$ ), which, according to current theories, are the phases when most of the mass locked up in current black holes was accreted (e.g., Hopkins \& Hernquist 2009; Treister et al. 2012).

Wide-area surveys have existed for years at optical, infrared, and radio wavelengths, but have only recently been underway in X-rays at energies above $2 \mathrm{keV}$ and at depths capable of pushing to cosmological distances. While the deep, small area Chandra Deep Field South Survey $\left(0.13 \mathrm{deg}^{2}\right.$; Giacconi et al. 2001; Xue et al. 2011) has uncovered the faintest AGNs and has entered the flux regime where the number density of non-active galaxies surpasses that of active systems (Lehmer et al. 2012), and medium-area surveys like XMM-Newton and Chandra-COSMOS (2.2 $\mathrm{deg}^{2}$; Cappelluti et al. 2007; Hasinger et al. 2007; Elvis et al. 2009; Brusa et al. 2010; Civano et al. 2012, 2015; Marchesi et al. 2015) have identified nearly 2,000 moderate-luminosity AGNs $\left(10^{43} \mathrm{erg} \mathrm{s}^{-1}<L_{x}<\right.$ $10^{44} \mathrm{erg} \mathrm{s}^{-1}$ ), the $L_{x}>10^{45} \mathrm{erg} \mathrm{s}^{-1}$ population has been a missing tier in our hard X-ray census of supermassive black hole growth. This population began to be revealed with larger area $\left(\sim 10 \mathrm{deg}^{2}\right)$ surveys, such as XBoötes $\left(9 \mathrm{deg}^{2}\right.$; Kenter et al. 2005; Murray et al. 2005) and the Chandra Multi-wavelength Project (ChaMP, $10 \mathrm{deg}^{2}$; Kim et al. 2007), as well as the more recent XMM-Newton survey in the Herschel ATLAS field (7.1 $\mathrm{deg}^{2}$; Ranalli et al. 2015). The advent of the widest-area surveys $\left(>15 \mathrm{deg}^{2}\right)$, including the "Stripe 82X" survey (LaMassa et al. 2013a, 2013b), which, as we discuss below, now reaches $\sim 31.3 \mathrm{deg}^{2}$, as well as the $50 \mathrm{deg}^{2} X M M-X X L$ (PI: Pierre) and the $\sim 877 \mathrm{deg}^{2} X M M$-Serendipitous (Rosen et al. 2015) surveys, provides a chance to study the evolution of the most luminous AGN in unprecedented detail. However, though the $X M M$-Serendipitous survey covers an order of magnitude more area than the dedicated large-area XMMNewton surveys, an important component is missing: supporting multi-wavelength data which allows the X-ray photons to be identified with discrete sources and the properties of these objects to be characterized. A field which contains such supporting information, such as the Sloan Digital Sky Survey (SDSS; York et al. 2000) Stripe 82 region, is therefore an ideal location to execute an X-ray survey with maximal efficiency for returning comprehensive results.

Stripe 82 is a $300 \mathrm{deg}^{2}$ equatorial region imaged between 80 and 120 times as part of a supernova survey with SDSS (Frieman et al. 2008). The coadded photometry reaches 1.2-2.2 magnitudes deeper than any single SDSS scan $(r \sim 24.6$ versus $r \sim 22.2$; Annis et al. 2014; Jiang et al. 2014), and the full field has existing optical spectroscopy from SDSS and SDSS BOSS (Data Releases 9 and 10; Ahn et al. 2012, 2014), 2 SLAQ (Croom et al. 2009), and WiggleZ (Drinkwater et al. 2010), with partial coverage from DEEP2 (Newman et al. 2013), PRIMUS (Coil et al. 2011), 6dF (Jones et al. 2004, 2009), the VIMOS VLT Deep Survey (VVDS Garilli et al. 2008), a deep spectroscopic survey of faint quasars from Jiang et al. (2006), and a pre-BOSS pilot survey using Hectospec on MMT (Ross et al. 2012). Existing multi-wavelength data in Stripe 82 include near-infrared observations from UKIDSS (Hewett et al. 2006; Casali et al. 2007; Lawrence et al. 2007) and the
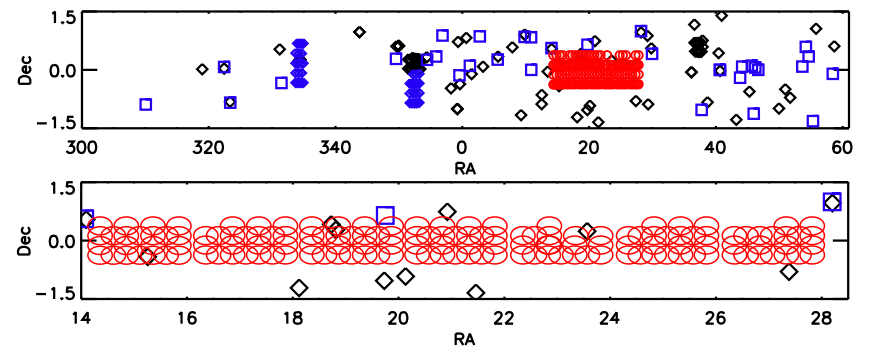

Figure 1. Distribution of archival Chandra observations (black diamonds), archival XMM-Newton observations (blue squares), XMM-Newton AO10 observations (blue diamonds), and XMM-Newton AO13 observations (red circles) for the full Stripe 82 region (top) and the XMM-Newton AO13 area (bottom). The symbol size is to scale with the field of view for the AO13 pointings in the bottom panel only.

VISTA Hemisphere Survey (VHS; McMahon et al. 2013); farinfrared coverage from Herschel over $79 \mathrm{deg}^{2}$ (Viero et al. 2014); ultraviolet coverage with GALEX (Morrissey et al. 2007); radio observations at $1.4 \mathrm{GHz}$ with Faint Images of the Radio Sky at Twenty centimeters (FIRST) (Becker et al. 1995; White et al. 1997; Becker et al. 2012; Helfand et al. 2015), with deeper VLA coverage over $80 \mathrm{deg}^{2}$ (Hodge et al. 2011); and millimeter observations with the Atacama Cosmology Telescope (ACT; Fowler et al. 2007; Swetz et al. 2011). Additionally, there is Spitzer coverage in the field from the Spitzer-HETDEX Exploratory Large Area survey over $28 \mathrm{deg}^{2}$ (SHELA; PI: C. Papovich) and the Spitzer IRAC Equatorial Survey over $110 \mathrm{deg}^{2}$ (SpIES; PI: G. Richards; J. Timlin et al. 2015, in preparation), deeper near-infrared $J$ and $K$ band coverage, to limits of $22 \mathrm{mag}$ (AB), from the VISTACFHT Stripe 82 Survey over $140 \mathrm{deg}^{2}$ (VICS82, PIs: Geach, Lin, Makler; J. Geach et al. 2015, in preparation), and midinfrared coverage from the all-sky WISE mission (Wright et al. 2010).

To take advantage of this rich multi-wavelength coverage, we designed the wide-area Stripe $82 \mathrm{X}$ survey (LaMassa et al. 2013a, 2013b) to uncover a representative population of rare, high-luminosity AGNs at high redshift. Here we release the next installment of the Stripe $82 \mathrm{X}$ point-source catalog, which includes data awarded to our team in response to $X M M$ Newton Announcement Opportunity 13 ("AO13”), representing $\sim 980 \mathrm{ks}$ of observing time (PI: C. M. Urry; Proposal ID 074283). We also publish updated catalogs from our previous Stripe $82 \mathrm{X}$ data releases from archival Chandra and XMM-Newton observations in Stripe 82 (LaMassa et al. 2013a, 2013b) and a pilot XMM-Newton program granted to our team in AO10 (PI: C. M. Urry; LaMassa et al. 2013a). The positions of the X-ray pointings used in Stripe $82 \mathrm{X}$ are shown in Figure 1.

In Section 2, we discuss the data analysis for XMM-Newton AO13, which we then combine with the previously released Chandra and XMM-Newton data in Section 3 to characterize the Stripe 82 X-ray survey to date, currently spanning $\sim 31.3 \mathrm{deg}^{2}$ of non-overlapping area. In Section 4 , we match the X-ray source lists to publicly available catalogs from SDSS, WISE, UKIDSS, VHS, GALEX, FIRST, and Herschel. Throughout, we adopt a cosmology of $H_{0}=70 \mathrm{~km} \mathrm{~s}^{-1} \mathrm{Mpc}^{-1}$, $\Omega_{M}=0.27$, and $\Lambda=0.73$. 
Table 1

XMM-Newton AO13 Observation Summary

\begin{tabular}{|c|c|c|c|c|c|}
\hline ObsID & Observation Date & Center R.A. & Center decl. & Discarded Pseudo-exposures & Area $\left(\mathrm{deg}^{2}\right)$ \\
\hline 0742830101 & 2014 Jul & $00: 57: 23.99$ & $-00: 22: 30.0$ & .. & 2.33 \\
\hline 0747390101 & $2014 \mathrm{Jul}$ & 01:05:23.99 & $-00: 22: 30.0$ & $22(\mathrm{PN}, \mathrm{M} 1, \mathrm{M} 2)$ & 2.22 \\
\hline 0747400101 & 2014 Jul & $01: 13: 24.00$ & $-00: 22: 30.0$ & $\ldots$ & 2.33 \\
\hline 0747410101 & 2015 Jan & $01: 21: 24.00$ & $-00: 22: 30.0$ & $6(\mathrm{PN}), 8(\mathrm{PN}), 13(\mathrm{PN})$ & 2.32 \\
\hline 0747420101 & 2015 Jan & 01:29:23.99 & $-00: 22: 30.0$ & $\begin{array}{l}16 \text { (PN, M1, M2), } 18 \text { (PN, M1, M2) } \\
20 \text { (PN, M1, M2), } 21 \text { (PN, M1, M2) }\end{array}$ & 1.95 \\
\hline 0747430101 & 2014 Jul & $01: 37: 23.99$ & $-00: 22: 30.0$ & $22(\mathrm{PN}, \mathrm{M} 1, \mathrm{M} 2)$ & 2.22 \\
\hline 0747440101 & 2014 Aug & $01: 45: 23.99$ & $-00: 22: 30.0$ & 22 (PN, M1, M2) & 2.22 \\
\hline
\end{tabular}

\section{XMM-Newton AO13 OBSERVATIONS AND DATA ANALYSIS}

Our XMM-Newton AO13 program was executed between 2014 July and 2015 January in a series of seven observations, as summarized in Table 1. Each observation consists of 22 individual pointings, or pseudo-exposures, which were carried out in "mosaic mode." This observing mode efficiently surveys a large area with individual pointings that have relatively short exposure times. To reduce overhead, the EPIC offset tables are only uploaded (for the MOS detectors) and calculated (for the PN detector) for the first pointing in the series. In our AO13 program, each pseudo-exposure is separated by a half field of view $\left(\sim 15^{\prime}\right)$ to enable a greater depth to be achieved in the overlapping regions. The median exposure time for individual pointings before filtering is $\sim 5.2 \mathrm{ks}$ for MOS1 and MOS2 and $\sim 4.7 \mathrm{ks}$ for PN, while the coadded depth in the overlapping observations reaches $\sim 6-8 \mathrm{ks}$ after filtering and correcting for vignetting (i.e., the energy-dependent decrease in effective area with off-axis distance).

The observational data files (ODF) were generated using the Science Analysis System (SAS) tasks (HEASOFT v. 6.16) emproc and epproc to create the MOS1, MOS2, PN, and PN out-of-time (OoT) events files. The OoT events occur from photons that are detected during CCD readout and recorded at random positions along the readout column. This effect is most significant for the PN detector and affects $\sim 6.3 \%$ of the observing time. The PN images can be statistically corrected for this effect using the PN OoT files.

The mosaicked observations were separated into individual pseudo-exposures using the SAS package emosaic_prep. Each pseudo-exposure was then filtered as described below.

\subsection{Flare Filtering}

Episodes of high levels of background radiation cause flaring in the XMM-Newton events files, hampering signal detection amidst the noise. To create good time intervals (GTIs), i.e., selecting events from observation periods where flaring is minimal, we started with a statistical approach. We created histograms of the count rate at high energies, $10-12 \mathrm{keV}$ for the MOS detectors and 10-14 keV for the PN detector, in time bins of $100 \mathrm{~s}$, extracted from single events (PATTERN $==0$ ). We created GTIs by excluding periods where the count rate was $\geqslant 3 \sigma$ above the mean and applied this filtering to the events file. From this events file, we searched for periods of low-energy $(0.3-10 \mathrm{keV})$ flares, created GTIs from time bins where the count rate was below $3 \sigma$ of the mean, and applied this GTI file to the original events file.
While this method produced cleaned events files for most of the pseudo-exposures, it did a poor job in instances of intense flaring: a $3 \sigma$-clipping was inadequate since the count rate distributions have an extended tail within the $3 \sigma$ tolerance level. For these pointings, we inspected the count rate distributions by eye to determine a cut-off value to remove the tail of this distribution, visually inspecting both sets of GTIfiltered events files to assess which filtering best removed the background to enhance signal from the sources.

Finally, we note that some pseudo-exposures were badly hampered by flaring such that no GTI filtering could recover useful signal. In Table 1, we note which pseudo-exposures were subsequently discarded from our analysis, and whether this affected just the PN detector or all three detectors. We also indicate the effective area covered by each observation after removing flared pointings.

\subsection{Generating Products for Source Detection}

We extracted images from the GTI-filtered events files, using all valid events (PATTERN 0 to 12) for MOS1 and MOS2 and single to double events (PATTERN 0 to 4) for PN. We excluded the energy range from 1.45 to $1.54 \mathrm{keV}$ to avoid the $\mathrm{Al} \mathrm{K} \alpha$ line $(1.48 \mathrm{keV})$ from the detector background. The PN detector also has background emission lines from $\mathrm{Cu}$ at $\sim 7.4$ and $\sim 8.0 \mathrm{keV}$, so we excluded the energy ranges from 7.2 to $7.6 \mathrm{keV}$ and 7.8 to $8.2 \mathrm{keV}$ when extracting PN images. To correct for the OoT events, the PN OoT images were scaled by 0.063 and subtracted from the PN images. We then extracted MOS and PN images in the standard soft $(0.5-2 \mathrm{keV})$, hard (2-10 keV), and full $(0.5-10 \mathrm{keV})$ energy ranges and coadded the images among the detectors.

Exposure maps, which quantify the effective exposure time at each pixel in the detector, accounting for vignetting, were generated with the SAS task eexpmap for each detector and energy range. Since vignetting is a strong function of energy, we spectrally weighted the exposure maps such that the mean effective energy inputted into eexpmap is determined by assuming a power-law model where $\Gamma=2.0$ in the soft band and $\Gamma=1.7$ for the hard and full bands (see Cappelluti et al. 2007). This spectral model was also used to calculate energy conversion factors (ECFs) to convert from count rates to flux, as summarized in Table 2 (for a discussion of how different assumptions for $\Gamma$ affect the derived ECF, see Loaring et al. 2005; Cappelluti et al. 2007; Ranalli et al. 2013). The exposure maps were coadded among the detectors, weighted by their ECFs.

As described in detail by LaMassa et al. (2013a), we used the algorithm presented in Cappelluti et al. (2007) to create background maps. In brief, a simple source detection was run 
Table 2

Energy Conversion Factors (ECFs) ${ }^{\mathrm{a}}$

\begin{tabular}{lcc}
\hline \hline Band & PN & MOS \\
\hline Soft $(0.5-2 \mathrm{keV})$ & 7.45 & 2.00 \\
Hard $(2-10 \mathrm{keV})$ & 1.22 & 0.45 \\
Full $(0.5-10 \mathrm{keV})$ & 3.26 & 0.97 \\
\hline
\end{tabular}

Note.

${ }^{a}$ ECFs in units of counts $\mathrm{s}^{-1} / 10^{-11} \mathrm{erg} \mathrm{cm}^{-2} \mathrm{~s}^{-1}$. These are based on a spectral model where $N_{\mathrm{H}}=3 \times 10^{20} \mathrm{~cm}^{-2}$ and $\Gamma=2.0$ in the soft band and $\Gamma=1.7$ in the hard and full bands. The PN ECF takes into account energy ranges that were masked out due to detector background line emission.

on each detector image in each energy band using the SAS task eboxdetect with a low detection probability (likemin $=4)$. The positions of these sources were masked out. The remaining emission results from unresolved cosmic X-ray sources and local particle and detector background. These components were modeled and fit as discussed in Cappelluti et al. (2007) and LaMassa et al. (2013a) to produce a background map for each detector and energy range. The resulting background maps were then coadded among the detectors.

Before importing these products into the source detection software, we updated the header keywords "RA_NOM," "DEC_NOM," "EXP_ID," and "INSTRUME" to common values among the pseudo-exposures for each observation: the SAS source detection software, when running on these files simultaneously, will fail if the pseudo-exposures do not have common WCS, exposure ID, and instrument values. However, the "RA_PNT" and "DEC_PNT" header keywords were manually updated to reflect the central coordinates of each pseudo-exposure so that the point spread function (PSF) is correctly calculated during source detection. Detector masks were created using the SAS program emask, which uses the exposure map as input to determine which pixels are active for source detection.

\subsection{Source Detection}

We produced a preliminary list of sources by running the SAS task eboxdetect in "map" mode. This is a sliding-box algorithm that is run on the coadded images, background maps, exposure maps, and detector masks, where source counts are detected in a $5 \times 5$ pixel box with a low-probability threshold (likemin $=4$ ). This list is then used as an input into emldetect, which performs a maximum likelihood PSF fit to the eboxdetect sources. We used a minimum likelihood threshold $\left(\right.$ det_ml) of 6 , where det_ml $=-\ln P_{\text {random }}$, where $P_{\text {random }}$ is the Poissonian probability that a detection is due to random fluctuations. We also included a fit to mildly extended sources, where emldetect convolves the PSF with a $\beta$ model profile. We consider a source extended if the output extflag exceeds 0 . Finally, the ECFs reported in Table 2 were summed among the detectors included in the coadded pseudo-exposures (i.e., if the PN image was discarded due to flaring, the ECF sum is from the MOS detectors, while the PN ECF is included in the sum when all detectors are useable), such that emldetect reports the flux in physical units, as well as the count rates, for each detected source.

We ran the source detection algorithm separately for each observation. Due to the limited memory capabilities of the SAS source detection software, not all pseudo-exposures within an observation could be fit simultaneously. We therefore executed the source detection in batches, where adjacent columns in R. A. were fit simultaneously. To achieve the greatest coadded depth in the overlapping pointings, each column, other than the eastern and western edges of the mosaic, was included in two source detection runs. We note that the deepest overlap regions are fitted with this source-detection method. The source detection was also run separately for the different energy bands: soft $(0.5-2 \mathrm{keV})$, hard $(2-10 \mathrm{keV})$, and full $(0.5-10 \mathrm{keV})$.

\subsection{Source List Generation}

From the above procedure, we have six source lists per energy band per observation. Each list contains duplicate detections of some sources due to the overlapping regions covered in consecutive source detection runs. To produce a clean X-ray source list for each observation, we removed these duplicate detections. Following the algorithm used by the XMM-Newton Serendipitous Source Catalog (Watson et al. 2009) to flag duplicate observations, we consider objects from source lists covering overlapping areas to be the same if the distance between them is less than $d_{\text {cutoff, where }}$ $d_{\text {cutoff }}=\min \quad\left(0.9 \times d_{\mathrm{nn} 1}, \quad 0.9 \times d_{\mathrm{nn} 2}, \quad 15^{\prime \prime}\right.$,

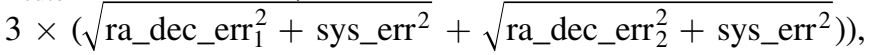
where $d_{\mathrm{nn} 1}\left(d_{\mathrm{nn} 2}\right)$ is the distance between the source and its nearest neighbor in the first (second) source list, ra_dec_err is the X-ray positional error reported by emldetect, and sys_err is a systematic positional error, taken to be $1^{\prime \prime}$, to account for the sources not having an external astrometric correction applied. A maximum search radius of $15^{\prime \prime}$ was chosen as the maximum cut-off distance based on simulations discussed in LaMassa et al. (2013a), where we found that this radius maximizes identification of output to input sources while minimizing spurious associations of distinct sources; due to the shallow nature of our observations, source confusion from a high density of unresolved sources is not a concern (see Section 3 for estimated source confusion rate). For duplicate detections of the same source, we retain the coordinates, flux, and count information for the object that has the highest detection probability, or det_ml. We perform this routine separately for each energy band, producing one clean source list per band.

We then merge these X-ray source lists for each energy band of an observation using the search criterion defined above to find matches among lists generated in the separate energy bands. If no match is found, the source is considered undetected in that band and its flux, flux error, counts, and det_ml are set to null while we retain this information for the band(s) where it is detected. While we have discarded sources that are extended in all bands in which they are detected, because the identification of clusters among the extended sources is in progress and will be reported later, we have flagged the sources that are point-like in one band and are extended in another band. The "ext_flag" is non-zero for these objects and is defined as follows: 1 -extended in the soft band, 2 - extended in the full band, 3 -extended in the hard band, 4 - extended in the soft and full bands, 5-extended in the soft and hard bands, 6-extended in the hard and full bands.

To produce the final catalog, the coordinates are averaged among the coordinates from the individual energy band catalogs and their positional errors are added in quadrature; we note that the significance of the detection is not taken into account when averaging the coordinates, but the uncertainty in 
the astrometric precision is included by adding the positional errors in quadrature. We then retain only objects where det_ml exceeds 15 (i.e., $>5 \sigma$ ) in at least one energy band (see Loaring et al. 2005; Mateos et al. 2008, for a discussion of det_ml limits and their effects on Eddington bias in the derived $\log N-\log S$ relation).

We caution that care must be taken when determining the reliability of the reported fluxes, as the catalog includes the emldetect reported fluxes for every band where the source was detected (i.e., det_ml $\geqslant 6$ ). Though the X-ray source can be considered a significant detection, as det_ $m l$ has to exceed 15 in at least one energy band for the source to be included in the catalog, the det_ml value for each band ought to be used to determine whether the reported flux is at an acceptable significance level. For reference, we use only fluxes in the subsequent analysis when det $_{-} m l \geqslant 15$ in that band.

Finally, we assign each X-ray source a unique record number ("rec_no"), ranging from 2359 to 5220, since the previous XMM-Newton Stripe $82 \mathrm{X}$ catalog release terminated at "rec_no" 2358. We also include columns "in_chandra" and "in_xmm" to note whether a source was detected in the archival Chandra or XMM-Newton Stripe 82X catalogs, respectively, as well as the corresponding identification number of the matched source; for the one XMM-Newton source that has two possible Chandra counterparts within the search radius (rec_no 3473), due to Chandra's superior spatial resolution, we list both of the Chandra matches. Details about each column are summarized in the Appendix.

\section{STRIPE 82X SURVEY SENSITIVITY AND LOG $N$-LOG $S$}

Similar to our previous Stripe $82 \mathrm{X}$ release, we gauge survey sensitivity for our XMM-Newton AO13 program via Monte Carlo simulations. For each observation, we generated a list of fluxes that follow published $\log N-\log S$ relations from $X M M$ COSMOS (Cappelluti et al. 2009) for the soft and hard bands and from ChaMP (Kim et al. 2007) for the full band. The minimum flux was set to 0.5 dex below the lowest detected flux in the source list for that observation and the maximum flux was set to $10^{-11} \mathrm{erg} \mathrm{s}^{-1} \mathrm{~cm}^{-2}$. An input source list is then generated by pulling random fluxes from this distribution which are then given random positions among the pseudoexposures making up the observation. We then use part of the simulator written for the XMM-Newton survey of CDFS (Ranalli et al. 2013) to convolve the input source list with the XMM-Newton PSF to create mock events files from which images were extracted. The observed background is then added to the simulated images. Since the exposure maps from the observations were used when generating the simulated events files and the observed background was added to the mock image, the simulations allow us to accurately gauge how well we can recover input sources given our observing conditions. Finally, we add Poissonian noise to the images and run these products through the source detection algorithm detailed above, using ancillary products (i.e., background maps, exposure maps, and detector masks) from the observations. We ran a suite of 20 simulations for each mosaicked observation.

Since we have both the input source list and the list of detected objects, we can estimate the spurious detection rate for our sample. We assume that any source detected above our det_ml threshold of 15 that does not have an input source within $15^{\prime \prime}$ is a spurious detection. We find our spurious detection rate for the XMM-Newton AO13 data to be $1.0 \%$, $0.67 \%$, and $0.33 \%$ in the soft, hard, and full bands, respectively. Furthermore, we can estimate the confusion fraction, which is when input sources are unresolved in the source detection and observed as one object. As we did in LaMassa et al. (2013a), we followed the prescription in Cappelluti et al. (2007) to test for source confusion, using the criterion $S_{\text {out }} /\left(S_{\text {in }}+3 \sigma_{\text {out }}\right)>1.5$, where $S_{\text {out }}$ is the output flux, $S_{\text {in }}$ is the input flux, and $\sigma_{\text {out }}$ is the emldetect-reported flux error. According to this metric, the source confusion rate is $0.15 \%, 0.10 \%$, and $0.16 \%$ percent in the soft, hard, and full bands, respectively.

To determine survey sensitivity, we generate histograms of all input fluxes and output fluxes for the det_ $m l \geqslant 15$ sources, and divide the latter by the former. We truncate this ratio where it reaches unity. By multiplying this sensitivity curve, which is a function of flux, by the survey area, we derive the area-flux curves shown in red in Figure 2. For comparison, we also plot the area-flux curves for the other components of the Stripe $82 \mathrm{X}$ survey in Figure 2: archival Chandra (green), archival XMMNewton (dark blue), and XMM-Newton AO10 (cyan). The black curve shows the total Stripe $82 X$ area-flux relation after removing overlapping observations between the Chandra and $X M M-N e w t o n$ surveys and between the XMM-Newton archival and AO13 surveys. To convert the observed $2-7 \mathrm{keV}$ and 0.5-7 keV Chandra bands to the XMM-Newton-defined hard and full bands of $2-10 \mathrm{keV}$ and $0.5-10 \mathrm{keV}$, we used the assumed power-law model of $\Gamma=1.7$ (see LaMassa et al. 2013a) to extrapolate the Chandra flux to the broader energy ranges (i.e., the hard and full fluxes were multiplied by factors of 1.36 and 1.21 , respectively).

In Table 3, we summarize the number of X-ray sources detected at a significant level for each Stripe $82 \mathrm{X}$ survey component. For the XMM-Newton surveys, a source is deemed significant if det_ml exceeds 15 in the specific energy band while for the Chandra survey, significance is determined by comparing the source flux at the pixel where it was detected with the $4.5 \sigma$ sensitivity map value at that pixel (see LaMassa et al. 2013b, for details). The "Total" row in Table 3 removes duplicate observations of the same source in overlapping pointings among the survey components. In the current $31.3 \mathrm{deg}^{2}$ Stripe $82 \mathrm{X}$ survey, 6181 distinct sources are significantly detected between XMM-Newton and Chandra.

We present the $\log N-\log S$ distribution, or number source density as a function of flux, of the current $31.3 \mathrm{deg}^{2}$ Stripe $82 \mathrm{X}$ survey in Figure 3. To be consistent with the area-flux curves, we combined the X-ray source lists from the archival Chandra, archival and AO10 XMM-Newton, and AO13 XMMNewton catalogs, removing all sources from observations that were discarded from the area-flux relation due to overlapping area. Targeted objects from archival observations were also removed as discussed in LaMassa et al. (2013a, 2013b). We also note that while the Chandra $\log N-\log S$ relation we published in LaMassa et al. (2013b) had the cluster fields removed a priori, we have made no such cut here since, as we mentioned in that work, we found that including or excluding such fields made no noticeable difference in the source density calculation. The Chandra hard and full band fluxes from the source list were converted from the $2-7 \mathrm{keV}$ and $0.5-7 \mathrm{keV}$ ranges to $2-10 \mathrm{keV}$ and $0.5-10 \mathrm{keV}$ bands as described above. For reference, we also plot the $\log N-\log S$ for a range of survey areas and depths: the deep, pencil-beam E-CDFS in the 

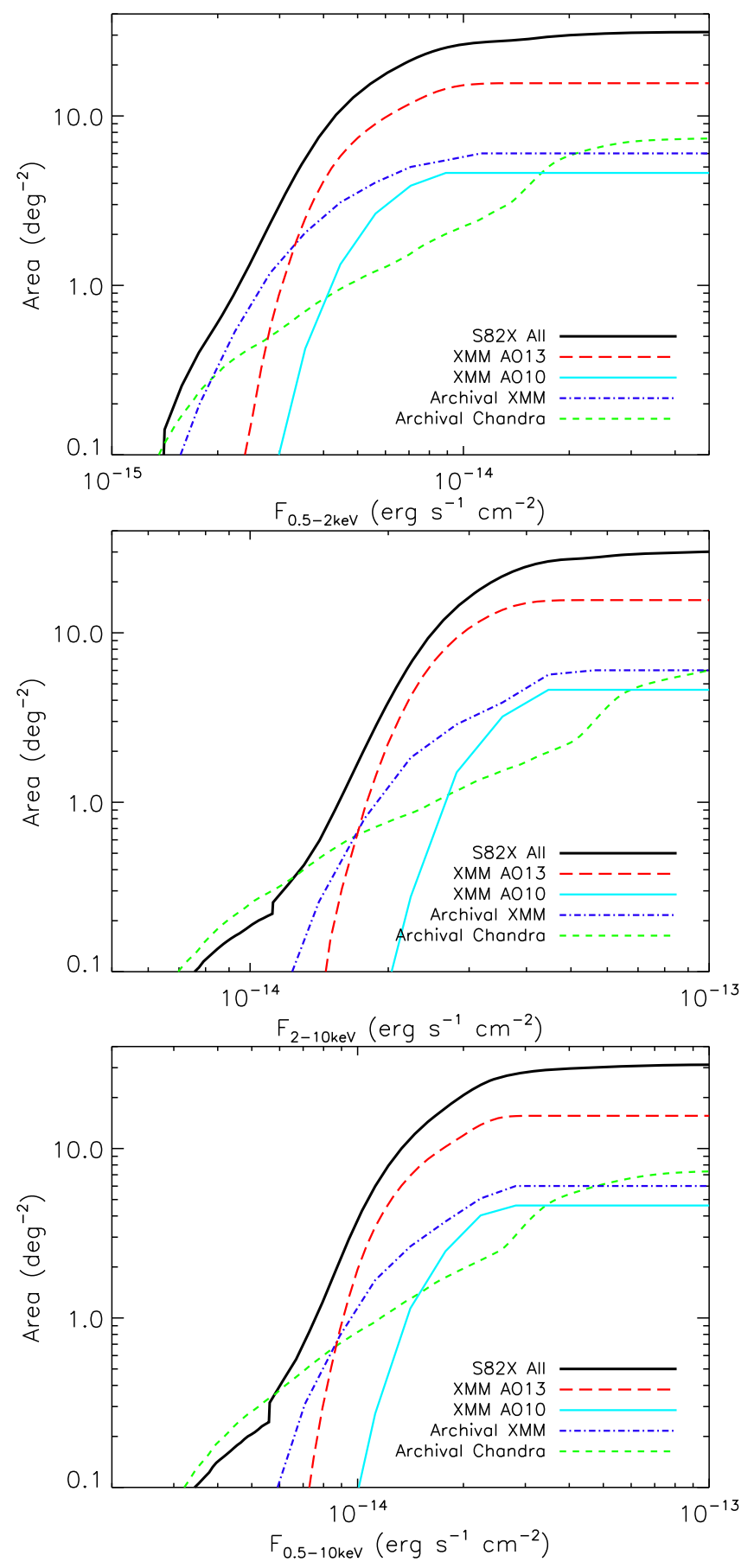

Figure 2. Area-flux curves for Stripe $82 \mathrm{X}$ in the soft (top), hard (middle), and full bands (bottom). While the colored curves show the full area for the individual data sets as indicated in the legends, the black curve illustrates the total area after removing observations from the archival Chandra and archival $X M M$-Newton surveys that overlap pointings from the XMM-Newton AO10 and/or AO13 surveys, and, in the case of the archival Chandra observations, archival XMM-Newton surveys; here, we have given preference to the widerarea coverage from XMM-Newton in overlapping pointings. Hence, deeper fluxes accessible by Chandra are consequently removed from the total Stripe $82 \mathrm{X}$ area-flux relation. The kink in the total area-flux curve in the hard and full bands comes from discontinuties induced by combining the individual areaflux curves from the archival pointings at lower flux limits.

soft band ( $0.3 \mathrm{deg}^{2}$; Lehmer et al. 2005) and the XMM-Newton survey of CDFS in the hard band $\left(\sim 0.25 \mathrm{deg}^{2}\right.$; Ranalli et al. 2013); the moderate-area, moderate-depth Chandra COSMOSLegacy Survey (2.2 $\mathrm{deg}^{2}$; Civano et al. 2015; Marchesi et al.
Table 3

X-ray Source Summary ${ }^{\mathrm{a}}$

\begin{tabular}{llrrr}
\hline \hline Survey & Soft & Hard & Full & Total \\
\hline Archival Chandra $\left(7.4 \mathrm{deg}^{2}\right)$ & 969 & 248 & 1137 & 1146 \\
Archival XMM-Newton $\left(6.0 \mathrm{deg}^{2}\right)$ & 1438 & 432 & 1411 & 1607 \\
XMM-Newton AO10 $\left(4.6 \mathrm{deg}^{2}\right)$ & 635 & 175 & 668 & 751 \\
XMM-Newton AO13 $\left(15.6 \mathrm{deg}^{2}\right)$ & 2440 & 715 & 2597 & 2862 \\
\hline Total $\left(31.3 \mathrm{deg}^{2}\right)^{\mathrm{d}}$ & 5150 & 1520 & 5628 & 6181 \\
\hline
\end{tabular}

Notes.

${ }^{a}$ The numbers correspond to the significant detections in each band. For Chandra, this is a $4.5 \sigma$ level based on comparing the flux with the sensitivity map (see LaMassa et al. 2013b, for details) and for the XMM-Newton surveys, the det_ml has to exceed 15 .

b The hard band spans $2-10 \mathrm{keV}$ for the XMM-Newton surveys but corresponds to $2-7 \mathrm{keV}$ for the Chandra survey.

${ }^{\mathrm{c}}$ The broad band is $0.5-10 \mathrm{keV}$ for the XMM-Newton surveys but ranges from $0.5-7 \mathrm{keV}$ for the Chandra survey.

${ }^{d}$ Duplicate observations of the same source and overlapping observations between surveys removed in total numbers.

2015) in all three bands; and the wide-area 2XMMi Serendipitous Survey in the soft and hard bands $\left(132 \mathrm{deg}^{2}\right.$; Mateos et al. 2008). The Stripe $82 X \log N-\log S$ agrees with the reported trends from other surveys in the soft-band, the high-flux end in the hard and full bands, and with CDFS at the low-flux end $\left(<2 \times 10^{-14} \mathrm{erg} \mathrm{s}^{-1}\right)$ in the hard band; discrepancies in these bands at lower fluxes (and between CDFS and COSMOS-Legacy and 2XMMi in the hard band at low fluxes) may be due to different methods for estimating survey sensitivity when generating area-flux curves and different assumed values for the power-law slope $(\Gamma)$ when converting count rate to flux, and are not necessarily atypical when comparing number counts from different surveys.

\section{MULTI-WAVELENGTH CATALOG MATCHING}

We searched for counterparts to the XMM-Newton AO13 sources in publicly available multi-wavelength databases: SDSS, WISE (Wright et al. 2010), UKIDSS (Hewett et al. 2006; Casali et al. 2007; Lawrence et al. 2007), VHS (McMahon et al. 2013), GALEX (Morrissey et al. 2007), FIRST, and the Herschel Survey of Stripe 82 (HerS; Viero et al. 2014). To determine whether a multi-wavelength association to an X-ray source represents the true astrophysical counterpart rather than a chance coincidence, we use the maximum likelihood estimator method (MLE; Sutherland \& Saunders 1992) to match between the $\mathrm{X}$-ray source lists and the ancillary catalogs. MLE takes into account the distance between an X-ray source and ancillary objects within the search radius, the astrometric errors of the $\mathrm{X}$-ray and ancillary sources, and the magnitude distribution of ancillary sources in the background to determine whether a potential multi-wavelength counterpart is more likely to be a background source or a true match. This method has been implemented in many X-ray surveys to identify reliable counterparts (e.g., Brusa et al. 2007, 2010; Cardamone et al. 2008; Laird et al. 2009; Luo et al. 2010; Civano et al. 2012; LaMassa et al. 2013a; Marchesi et al. 2015).

Ancillary objects within the search radius $\left(r_{\text {search }}\right)$, which is set at $7^{\prime \prime}$ for the XMM-Newton AO13 sources (see Brusa et al. 2010; LaMassa et al. 2013a), are assigned a likelihood ratio $(L R)$, which is the probability that the correct counterpart 

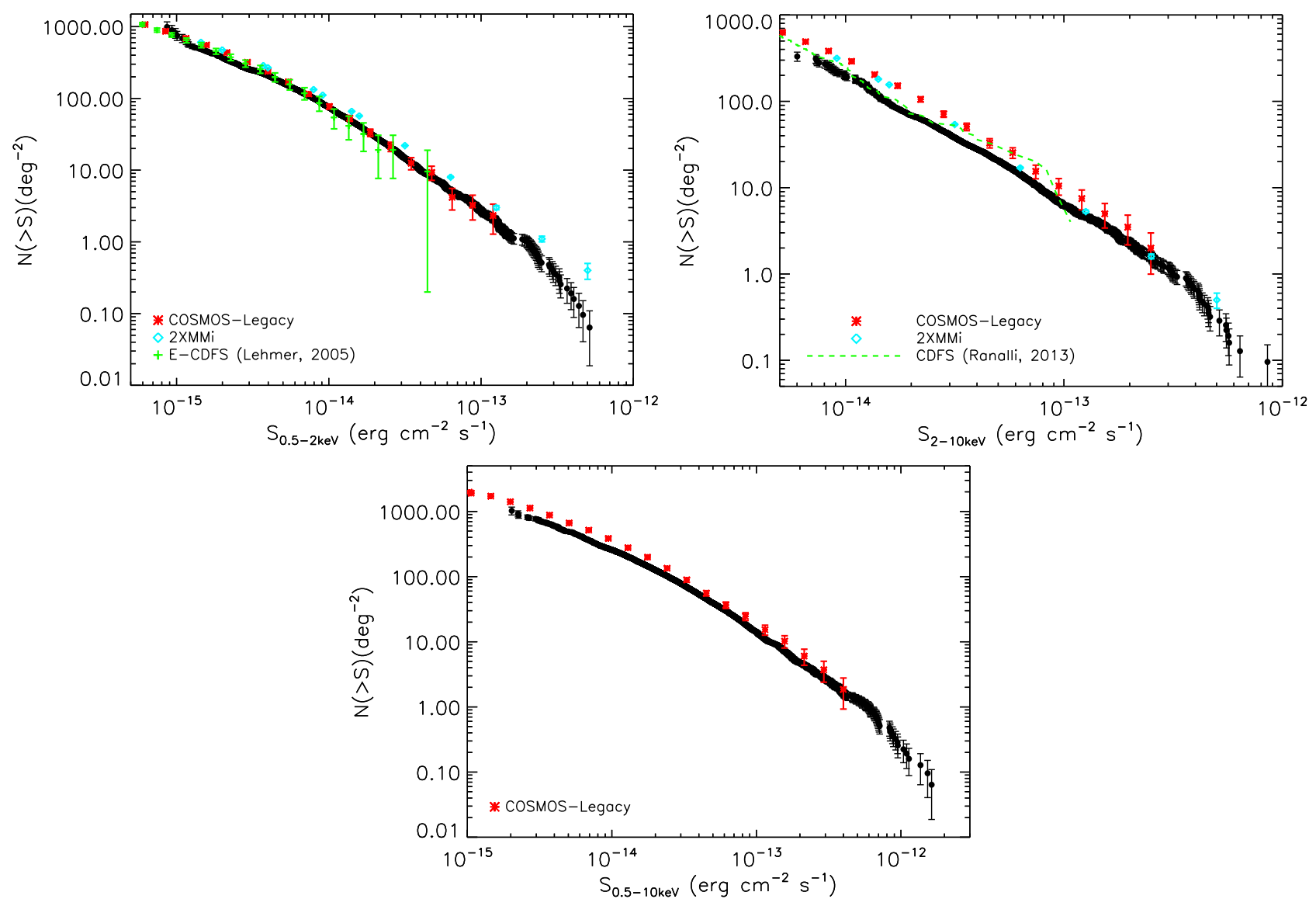

Figure 3. Cumulative $\log N$-Log $S$ relationship for the Stripe $82 \mathrm{X}$ survey (black circles) in the soft (top left), hard (top right), and full (bottom) bands. For reference, we also plot the source number density for other surveys, spanning the gamut from deep, pencil-beam surveys (i.e., the $0.3 \mathrm{deg}^{2} \mathrm{ECDF}-\mathrm{S}$ and $\sim 0.25 \mathrm{deg}^{2} \mathrm{CDFS}$; Lehmer et al. 2005; Ranalli et al. 2013, respectively), to a moderate-area, moderate depth survey (the 2.2 deg $^{2}$ Chandra COSMOS-Legacy; Civano et al. 2015; Marchesi et al. 2015), and a wide-area survey (the $132 \mathrm{deg}^{2}$ 2XMMi Serendipitous Survey; Mateos et al. 2008).

is found within $r_{\text {search }}$ divided by the probability that a background ancillary source is there by chance:

$$
L R=\frac{q(m) f(r)}{n(m)}
$$

Here, $q(m)$ is the expected normalized magnitude distribution of counterparts within $r_{\text {search }}$ which is estimated by subtracting the histogram of sources found within the search radius from the histogram of background objects, where each histogram is normalized by the relevant search areas; $f(r)$ is the probability distribution of the astrometric errors ${ }^{23}$; and $n(m)$ is the normalized magnitude distribution of sources in the background. The background sources are taken as the objects found in an annulus around each X-ray source with an inner radius of $10^{\prime \prime}$ and an outer radius of $45^{\prime \prime}$; thus, sources that are potential counterparts, i.e., within $r_{\text {search }}$, are removed from the background estimation (Brusa et al. 2007). We note that the positional error for the X-ray sources includes a 1 " systematic error added in quadrature to the emldetect reported positional error to account for the lack of an external astrometric correction. This systematic astrometric error was not included in the previous release of the Stripe $82 \mathrm{X}$ catalog, and we subsequently found that bright $\mathrm{X}$-ray sources tended to have

\footnotetext{
${ }^{23} f(r)$ is modeled as a two-dimensional Gaussian distribution where the X-ray and ancillary positional errors are added in quadrature.
}

their positional errors underestimated by emldetect, such that counterparts were missed by the matching algorithm even though visual inspection of the X-ray sources and ancillary objects revealed bright multi-wavelength objects that are likely true matches (see also Brusa et al. 2010). Adding the $1^{\prime \prime}$ systematic error recovered these associations. Accordingly, the archival XMM-Newton and AO10 catalogs published previously have been updated here.

From $L R$, a reliability value is then calculated for every source:

$$
R=\frac{L R}{\sum_{i}(L R)_{i}+(1-Q)},
$$

where $Q$ is the ratio of the number of $\mathrm{X}$-ray sources that have ancillary objects within the search radius divided by the total number of X-ray sources; the $L R$ sum is over every potential counterpart within the search radius of the $\mathrm{X}$-ray source. This calculation is performed independently for every waveband to which we match the $\mathrm{X}$-ray source list. We use $R$ as a way to distinguish between true counterparts and chance associations. For X-ray sources that have more than one possible association within $r_{\text {search, }}$ we retain the potential counterpart with the highest reliability. To determine the critical reliability threshold above which we consider an association the true counterpart $\left(R_{\text {crit }}\right)$, we followed the methodology in LaMassa et al. (2013a): 
we produced a catalog where we shifted the X-ray positions by random amounts and matched the multi-wavelength catalogs to these randomized positions. The resulting reliability distribution then gives us an estimate of the number of contaminating spurious associations above $R_{\text {crit }}$. We pick our $R_{\text {crit }}$ threshold by examining the reliability histograms of the "true" matches, i.e., the original X-ray catalog, and the "spurious" matches, i.e., the catalog with randomized positions, in bins of 0.05 to determine where the fraction of spurious matches is $\sim 10 \%$. That bin then becomes our threshold $R_{\text {crit }}$ value.

As always, matching the X-ray source lists to ancillary catalogs is a balancing act between minimizing contamination from unassociated sources and maximizing counterpart identification. It is unavoidable that some true counterparts will be missed and that spurious associations will be promoted as real matches. In Sections 4.2-4.7 below, we note the number of spurious matches, i.e., the number of X-ray sources with randomized positions meeting the $R_{\text {crit }}$ threshold, to the number of total matches from the original X-ray catalog above $R_{\text {crit }}$ to provide an estimate of the counterpart contamination. We also show in Figures 4-10 the cumulative distribution of counterpart and spurious association fraction as a function of $r_{\text {sep }}$, the distance between the X-ray and counterpart coordinates, for objects exceeding $R_{\text {crit }}$. We remind the reader, however, that in addition to the separation between the sources, the astrometric error on both the X-ray and counterpart coordinates, the magnitude of the potential counterpart, and magnitude distribution of background sources all contribute to the calculated reliability value reported in the published catalogs.

As the X-ray sources represent a menagerie of astronomical objects (stars, galaxies, obscured AGNs, and unobscured AGNs) they will have a range of spectral energy distributions and thus not have the same relative strength among all the wave-bands in each ancillary catalog. For example, heavily obscured AGNs are much brighter in the redder optical and infrared bands, and would have optical magnitudes in the bluer bands more consistent with background sources, or perhaps even be dropouts in these bands, while the converse is true for unobscured AGNs. We therefore match the X-ray source list separately to each band in the multi-wavelength catalogs, determine $R_{\text {crit }}$ independently for each passband, and then merge the individual lists where we report the maximum $R_{\text {crit }}$ values among the matches for that catalog. The only exception to this procedure for the MLE matching is WISE since the W1 band is the most sensitive filter; all WISE sources in Stripe 82 have detections in the $W 1$ band so we do not miss any objects by matching to $W 1$ only. A high level summary of the multiwavelength matches to the XMM-Newton AO 13 data is presented in the fifth column of Table 4.

\subsection{Cross-matches between X-Ray Catalogs}

For the X-ray sources that are repeated among the individual catalogs (archival Chandra, archival and AO10 XMM-Newton, and AO13 XMM-Newton catalogs), we checked their multiwavelength counterpart matches against each other. In most cases, these are consistent, but in some instances, a counterpart is not found for an X-ray source in one catalog yet is in another. This situation can arise due to differences in X-ray positions and positional errors between the individual sources lists, as well as the differences in the magnitude distribution of background sources. If a counterpart is found in one $\mathrm{X}$-ray
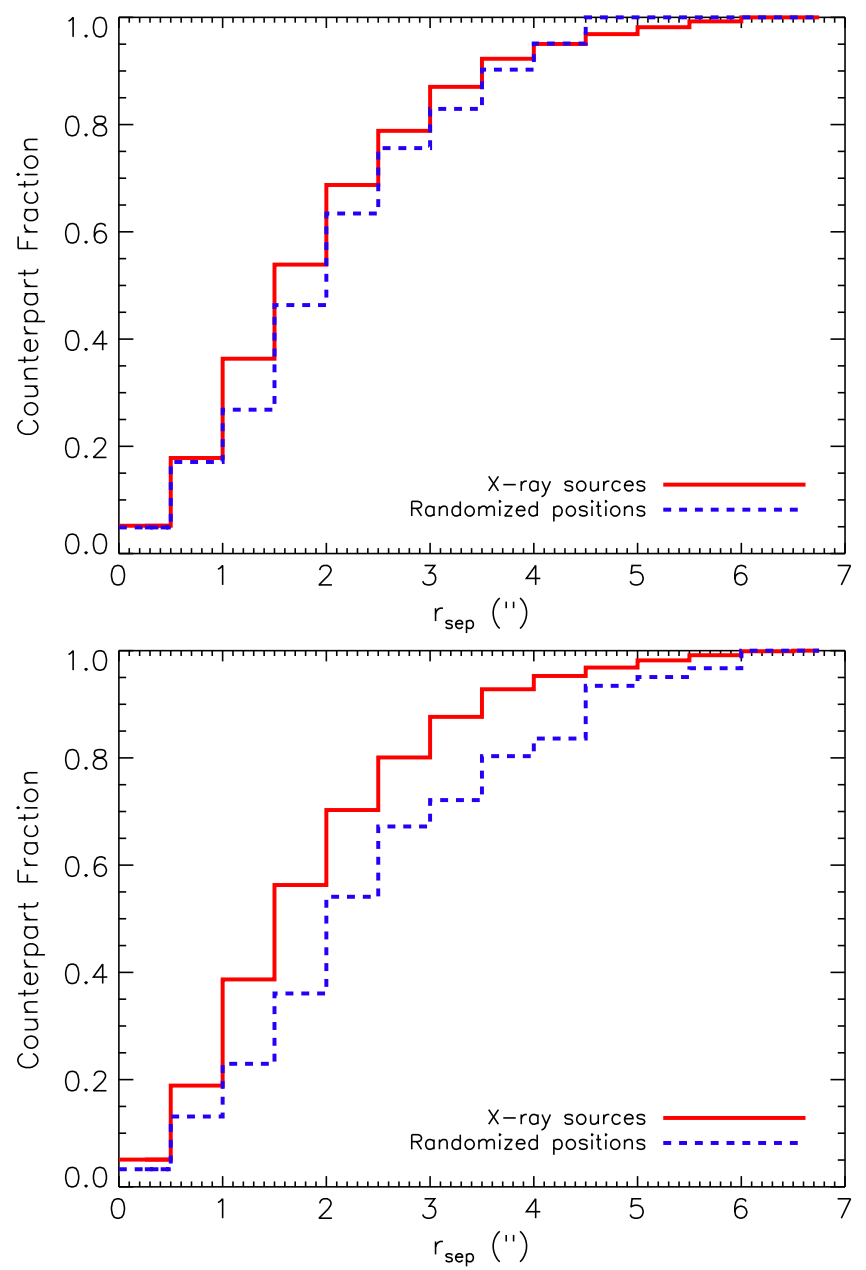

Figure 4. Cumulative distribution of the fraction of X-ray sources with an $r$ band counterpart above $R_{\text {crit }}$ as a function of distance between the X-ray and SDSS positions ( $r_{\text {sep }}$; red solid line) and between the randomized X-ray positions and SDSS sources (blue dashed line). The top panels are for the matches from single-epoch imaging (1852 X-ray/SDSS counterparts and 41 random matches) while the bottom panels show the matches to the coadded Jiang et al. (2014) catalog (1652 X-ray/coadded counterparts with 61 spurious associations). The number of spurious matches occurs at $r_{\text {sep }}$ distances similar to that as the un-shifted X-ray catalog, indicating that MLE helps to mitigate unassociated sources compared to nearest neighbor matching by using magnitude and astrometric precision information in the calculation.

catalog and not another, we promote that counterpart as a match in the latter catalog. To keep track of such promoted matches, we have included the following flags: "ch_cp_flag," "xmm_archive_cp_flag," and "xmm_ao13_cp_flag" to indicate which counterparts were promoted into that catalog based on MLE matching from the archival Chandra catalog, archival and AO10 XMM-Newton catalog, and AO13 XMM-Newton catalog, respectively. If these fields are empty, then the independent MLE matching to the individual catalogs gave consistent results. Otherwise, the following numbers indicate which multi-wavelength counterpart is the promoted match: 1 - SDSS counterpart found but photometry rejected for failing quality control checks; 2-SDSS; 3-redshift; 4-WISE counterpart found but rejected for failing quality control checks; 5-WISE; 6-UKIDSS; 7-VHS; 8-GALEX. ${ }^{24}$

\footnotetext{
${ }^{24}$ None of the UKIDSS or VHS matches were rejected for compromised photometry.
} 
The Astrophysical Journal, 817:172 (21pp), 2016 February 1

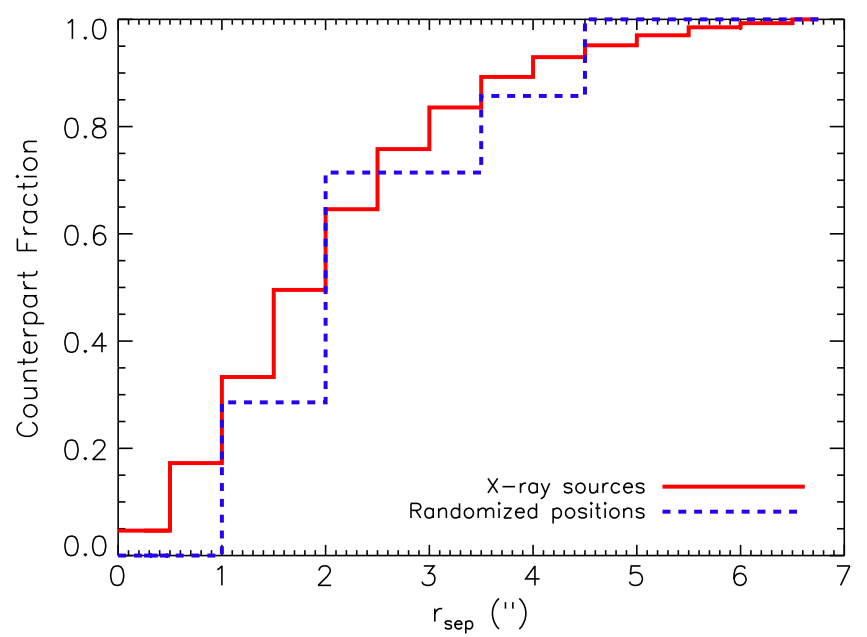

Figure 5. Similar to Figure 4, but for the X-ray/WISE matches to the $W 1$ band, where 2087 counterparts (seven spurious associations) are found (before discarding those failing quality control checks) above $R_{\text {crit. }}$.

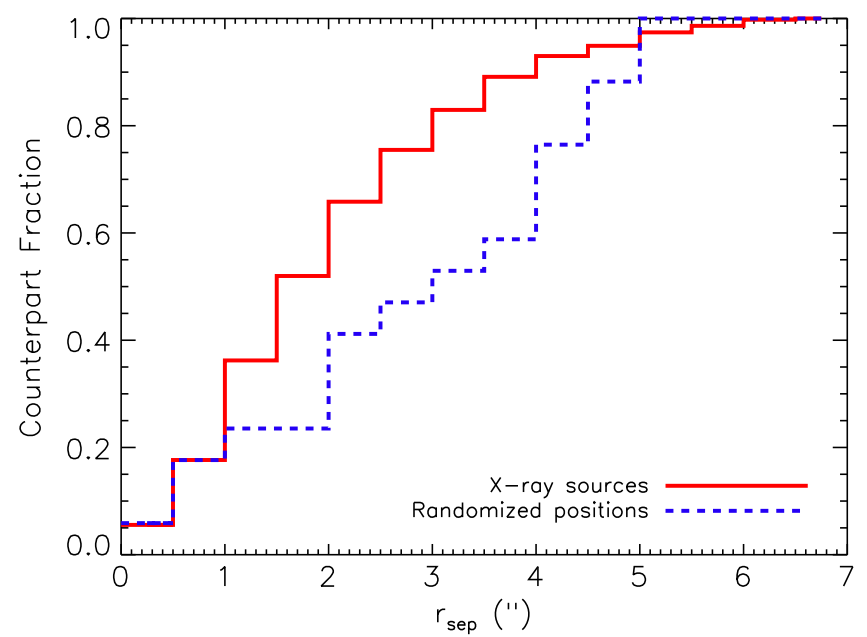

Figure 6. Similar to Figure 4, but for the X-ray/UKIDSS matches to the $K$ band, where 1314 counterparts and 17 matches to randomized positions are found above $R_{\text {crit }}$.

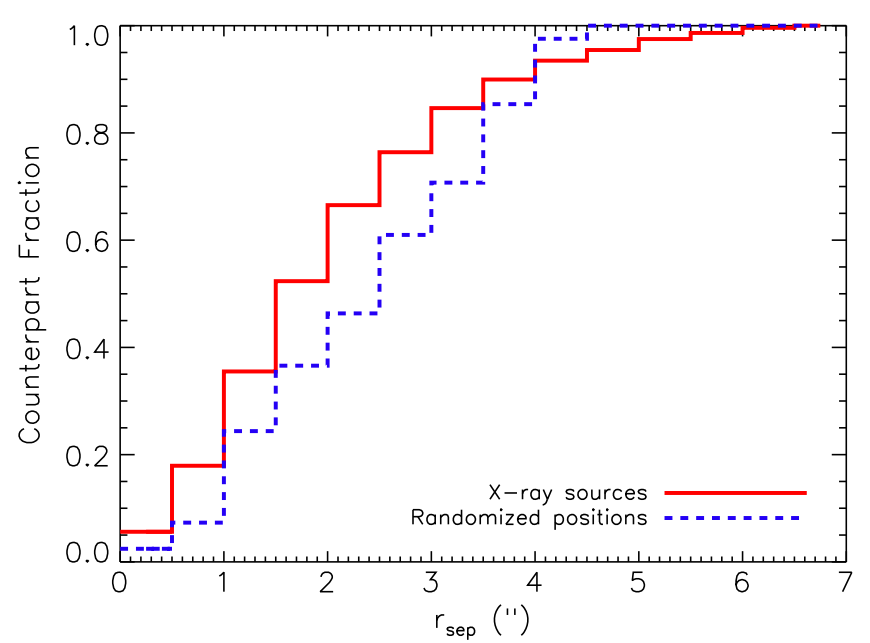

Figure 7. Similar to Figure 4, but for the X-ray/VHS matches to the $K$ band, where 1763 counterparts and 41 spurious associations are above $R_{\text {crit }}$.

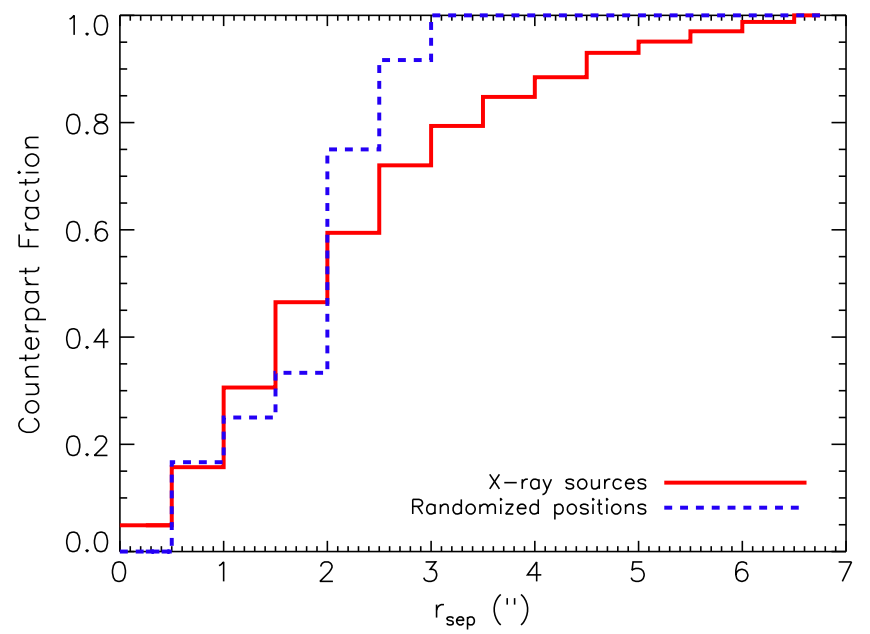

Figure 8. Similar to Figure 4, but for the X-ray/GALEX matches to the NUV band, where 572 counterpartsand 12 matches to randomized positions lie above $R_{\text {crit. }}$.

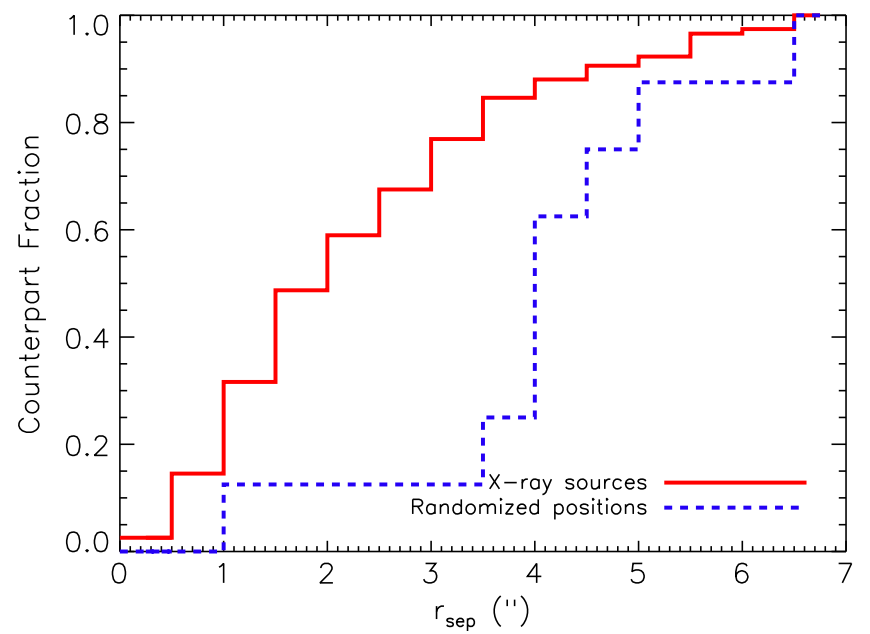

Figure 9. Similar to Figure 4, but for the X-ray and FIRST nearest-neighbor matches, with 116 counterparts and eight randomized matches found within $r_{\text {search }}=7^{\prime \prime}$. Here, many of the spurious associations are found at higher separation distances due to the low number density of radio and X-ray sources.

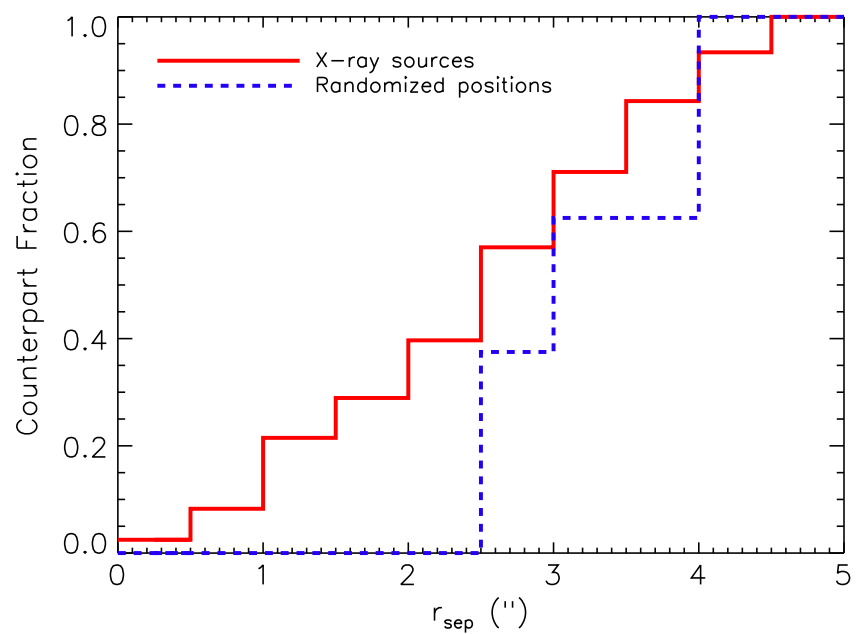

Figure 10. Similar to Figure 4, but for the X-ray and Herschel nearest-neighbor matches, with 121 counterparts and 8 spurious associations within $r_{\text {search }}=5^{\prime \prime}$. Just as Figure 9 shows, the matching between the X-ray source list and FIRST, most of the spurious matches occurs at higher values of $r_{\mathrm{sep}}$. 
Table 4

Multi-wavelength Counterpart Summary ${ }^{\mathrm{a}}$

\begin{tabular}{llrrrr}
\hline \hline Survey & $\begin{array}{l}\text { Chandra } \\
\text { Archival }\end{array}$ & $\begin{array}{r}\text { XMM- } \\
\text { Newton } \\
\text { Archival }\end{array}$ & $\begin{array}{r}\text { XMM- } \\
\text { Newton } \\
\text { AO10 }\end{array}$ & $\begin{array}{r}\text { XMM- } \\
\text { Newton } \\
\text { AO13 }\end{array}$ & Total $^{\mathrm{b}}$ \\
\hline SDSS $^{\mathrm{c}}$ & $874(118)$ & $1258(190)$ & $614(66)$ & 2438 & 5009 \\
& & & & $(178)$ & $(530)$ \\
WISE & 686 & 948 & 531 & 2033 & 4006 \\
UKIDSS & 568 & 923 & 503 & 1784 & 3643 \\
VHS & 610 & 995 & 518 & 2119 & 4093 \\
GALEX & 166 & 254 & 82 & 607 & 1080 \\
FIRST & 42 & 55 & 27 & 116 & 232 \\
Herschel & 9 & 9 & $\ldots$ & 121 & 133 \\
Redshifts & 339 & 465 & 292 & 828 & 1842 \\
\hline
\end{tabular}

Notes.

${ }^{\mathrm{a}}$ The counterpart numbers quoted in the text refer to associations found from matching the individual X-ray catalogs with the multi-wavelength source lists. Here, we include the final numbers that include "promoted" matches, found from cross-correlating the counterparts among the X-ray catalogs (see Sections 4.1 and A.7 for details).

${ }^{\mathrm{b}}$ Duplicate sources among surveys removed from total numbers.

${ }^{c}$ Includes matches to the single-epoch and coadded catalogs. The number of sources found in the coadded catalog that do not have matches in the singleepoch data are quoted in parentheses.

While the number of matches quoted in the text below refer to the sources above $R_{\text {crit }}$ in each catalog, the tally in the Table 4 include the promoted counterparts found from crossmatching the catalogs. The remainder of this section pertains to the multi-wavelength catalog matching to the XMM-Newton AO13 source list, while updates to the previous released Stripe $82 \mathrm{X}$ catalogs are discussed in the Appendix.

\section{2. $S D S S$}

We matched the X-ray sources to the separate $u, g, r, i$, and $z$ bands in the single-epoch SDSS photometry from Data Release 9 (Ahn et al. 2012, DR9), where a uniform 0"'1 error was assumed for the SDSS astrometry (Rots \& Budavári 2011). We imposed the following $R_{\text {crit }}$ values for the individual SDSS bands: $u-0.75, g-0.80, r-0.85, i-0.85, z-0.80$, with the estimated number of spurious association rate being 36/1989, 43/2006, 41/1852, 21/1819, and 51/1926, respectively. Figure 4 (top) shows the cumulative distribution of counterparts and spurious associations above the $r$-band $R_{\text {crit }}$ value as a function of distance between the X-ray and SDSS source.

We removed from these individual band source lists any SDSS object that did not exceed the $R_{\text {crit }}$ threshold, and then checked by eye the instances where more than one SDSS source is matched to an X-ray source to determine which optical source is the most likely counterpart. The preferred match is usually the SDSS source with the greatest number of matches among the individual bands and/or the brightest object. From our band-merged list, we then perform a photometric quality control to check for saturation, blending, or photometry that is not well measured. ${ }^{25}$ Objects that do not

\footnotetext{
${ }^{25}$ We report the photometry for objects that meet the follow requirements: (NOT_SATUR) OR (SATUR AND (NOT SATUR_CENTER)), (NOT BLENDED) OR (NOT NODEBLEND), (NOT BRIGHT) AND (NOT DEBLEND_TOO_MANY_PEAKS) AND (NOT PEAKCENTER) AND (NOT NOTCHECKED) AND (NOT NOPROFILE). An object that failed any of these quality control checks has the photometry set to -999 in the catalog.
}

meet these requirements are flagged in the "SDSS_rej" column as "yes" in the catalog, though we retain the SDSS coordinates and ObjID to note that these sources are optically detected even if the photometry is compromised. Finally, we check the remaining images by eye to remove optical artifacts, such as diffraction spikes and noise due to emission from nearby bright objects.

We then matched the full X-ray catalog to the coadded SDSS source lists presented in Jiang et al. (2014), which are 1.9-2.2 mag deeper than the single-epoch SDSS imaging, with $5 \sigma$ magnitude limits of 23.9, 25.1, 24.6, 24.1, and 22.8 (AB) in the $u, g, r, i$, and $z$ bands. Here, we utilize the mag_auto fields returned by SExtractor (Bertin \& Arnouts 1996) for the MLE algorithm. Jiang et al. (2014) performed the image coaddition by separating each of the 12 SDSS parallel scans that cover Stripe 82 into 401 individual regions, extracting aperture magnitudes separately for each of the five bands. They then provide 24,060 individual catalogs, where each band, region, and scan line are independent catalogs, which can include duplicate observations of the same source among these catalogs that cover adjacent area. Thus, we first produced "cleaned" SDSS coadded catalogs by only retaining objects within $45^{\prime \prime}$ of the XMM-Newton AO13 sources, since these are the data we need to estimate the background and find counterparts. We then search for duplicate observations within each band by searching for matches within 0.15 , retaining the coordinates and photometry for the object that has the highest signal-tonoise. We match the X-ray sources to each of these cleaned coadded catalogs. Here, the astrometric errors in the coadded images are similar to those of the single-epoch images due to the method used when generating the coadds (Jiang et al. 2014). However, we conservatively used a value of 0 !" 2 based on observed positional offsets between SDSS coadded sources and FIRST objects (I. McGreer 2015, private communication). We find the following $R_{\text {crit }}$ cut-offs: $u-$ $0.85, g-0.9, r-0.9, i-0.9, z-0.85$, with the spurious association rate being 20/1799, 41/1751, 61/1652, 40/1530, and $37 / 1816$, respectively; the cumulative fraction of matches as a function of $r_{\text {search }}$ above $R_{\text {crit }}$ for both the X-ray source list and randomized positions is shown in the bottom panel of Figure 4 . We note that the lower number of sources here compared with the single-epoch imaging data is due to the higher reliability thresholds we impose for the coadded catalog. However, the number of spurious associations in the lower reliability bins becomes a much higher fraction of the total number of true X-ray sources in those bins, so we have erred on the side of caution to minimize the number of random associations in our sample.

From these counterparts found from matching to the coadded images, we keep only the sources that do not have a counterpart in the single-epoch imaging. Since Jiang et al. (2014) do not provide a band-matched catalog or cross-identify the same source among the multiple-bands, we consider an optical source to be the same object if it is within $\sim 0$ " 6 with no other object found in that band within $1^{\prime \prime}$; if no match in another band is found meeting these requirements, the source is assumed to be a drop-out in that band. The reported SDSS coordinates are the average of the coordinates in the individual band catalogs where the source is detected. The objects found from the coadded catalog are marked in the "SDSS_coadd" column as "yes." 
In total, we find SDSS counterparts for $2438 \mathrm{X}$-ray sources ( $85 \%$ of the sample), 178 of which are not found in the singleepoch SDSS imaging but are detected in the coadded catalog, and as expected are generally fainter. We list the information for the SDSS counterparts found from the single-epoch catalog, where available, to allow the user to easily query the main SDSS database to fetch relevant information using the unique SDSS ObjID or SDSS coordinates; similar data, such as aperture magnitudes and errors, from the coadded Jiang et al. (2014) catalog would involve querying 24,060 individual catalogs, while such data are linked in the main SDSS database.

\subsubsection{Optical Spectra}

We mined the following public spectroscopic catalogs to find redshifts, and where possible, optical classifications of the X-ray sources with SDSS counterparts: SDSS Data Release 12 (DR12; Alam et al. 2015), 2SLAQ (Croom et al. 2009), preBOSS pilot survey using Hectospec on MMT (Ross et al. 2012), and 6dF (Jones et al. 2004, 2009). We checked by eye the 41 spectra that had the zwarning flag set by the SDSS pipeline. While we were able to verify some of these redshifts, we were not able to find a reliable redshift solution for 26 of these objects, and consequently set their redshifts to zero in the catalog. We also obtained spectra for 12 and 6 sources in 2014 September and 2015 January, respectively, through our dedicated follow-up program with WIYN HYDRA; the spectra were reduced with the IRAF task dohydra where we identified redshifts based on emission and/or absorption features, or classified stars on the basis of their rest-frame absorption and emission lines. About $29 \%$ of the X-ray sources (828 objects) have secure redshifts. The calculation of photometric redshifts for the remainder of the sources is underway (T. Ananna et al. 2015 , in preparation).

The databases we mined provide an automatic classification of sources based on their optical spectra, where "QSO"s or "AGNs" are objects that have at least one broad emission line in their spectra (generally a full-width half max exceeding $2000 \mathrm{~km} \mathrm{~s}^{-1}$ ). Sources lacking broad emission lines are classified as "galaxies," where this type includes objects with narrow emission lines (Type 2 and elusive AGNs, i.e. those objects with emission line ratios consistent with star-forming galaxies; Baldwin et al. 1981; Maiolino et al. 2003), absorption lines only, and even blazars with featureless optical spectra that are not flagged as active galaxies by optical spectroscopic pipelines. We have followed this methodology when classifying sources from our spectroscopic follow-up campaign, where we reserve the class QSO to refer to broad-line objects and galaxies for sources lacking broad-lines. Stars are identified by emission and absorption transitions in their optical spectra.

\subsection{WISE}

Since publishing our initial Stripe $82 \mathrm{X}$ multi-wavelength matched catalogs in LaMassa et al. (2013a), the AllWISE Source Catalog was released, combining data from the cryogenic and NEOWISE missions (Wright et al. 2010; Mainzer et al. 2011). As this catalog has enhanced sensitivity and astrometric precision, we match the XMM-Newton AO13 X-ray source list to this release, and update the archival Chandra and XMM-Newton and XMM-Newton AO10 matches to AllWISE, as detailed in the appendix.
When doing the MLE matching to the $W 1$ band, using the "w1mpro" magnitude measured via profile-fitting photometry, we use a $R_{\text {crit }}$ of 0.9 , with seven spurious associations out of 2087 matches (see Figure 5). We then impose photometry control checks on the WISE sources, following our prescription in LaMassa et al. (2013a). We null out the magnitude in any band that was saturated (i.e., the fraction of saturated pixels, "wnsat" exceeds 0.05 , where $n$ refers to the band number); is likely a spurious detection associated with artifacts such as diffraction spikes, persistence, scattered light from nearby bright sources (i.e., if the "cc_flag" is non-zero); or moon level contamination (i.e., if "moon_lev" $\geqslant 5$, where "moon_lev" is the ratio of frames affected by scattered moonlight to the total number of frames and spans from 0 to 9). We also isolate extended sources, as their "wnmpro" magnitudes would be unreliable. These sources have the "ext_flag" set to non-zero. For these objects, we downloaded their elliptical photometry magnitudes ("wngmag") and discarded their photometry if their extended photometry magnitude flags were non-null. If a matched WISE source has photometry that fails the point-like or extended photometry quality checks in all bands, then the "wise_rej" flag is set to "yes" in the catalog and the associated photometry and coordinates are not reported.

Of the 2087 matched sources, 2031 (71\% of the XMMNewton AO13 sources) passed the quality assurance tests above. All the rejected sources were extended. Ten extended sources had non-flagged elliptical magnitude measurements and are marked with the "wise_ext" flag set to "yes" in the catalog.

\subsection{Near-infrared}

The XMM-Newton AO13 source list was matched independently to the near-infrared (NIR) catalogs from the UKIDSS Large Area Survey (LAS; Hewett et al. 2006; Casali et al. 2007; Lawrence et al. 2007; Warren et al. 2007) and VHS (McMahon et al. 2013). From both catalogs, we chose primary objects from the database ${ }^{26}$ and eliminated objects that were consistent with noise, i.e., "mergedclass" set to zero and "pnoise" $>0.05$. The magnitudes presented in the catalog are the "apermag3" values from the UKIDSS LAS and VHS databases, which are aperture-corrected magnitudes, with a $2^{\prime \prime}$ diameter aperture.

We matched the XMM-Newton AO13 source list to Data Release 8 of the UKIDSS LAS survey. Matching separately to the $Y(0.97-10.07 \mu \mathrm{m}), J(1.17-1.33 \mu \mathrm{m}), H(1.49-1.78 \mu \mathrm{m})$, and $K(2.03-2.37 \mu \mathrm{m})$ bands, we find $R_{\text {crit }}$ values of $0.75,0.85$, $0.75,0.75$, respectively, with a spurious association rate of $21 / 1375,15 / 1070,18 / 1335$, and $17 / 1314$, respectively (see Figure 6). When merging the separate lists together, we find a total of 1784 near-infrared counterparts, or $62 \%$ of the X-ray sample. We performed quality control checks on the photometry as explained in LaMassa et al. (2013a) to check for saturation, but no objects were flagged as being possibly saturated.

We used Data Release 3 of the VHS survey to match to the XMM-Newton AO13 catalog, where we adopt an astrometric uncertainty of 0 ". 14 for the VHS sources. VHS has coverage over Stripe 82 in the $J, H$, and $K$ bands, where we impose $R_{\text {crit }}$ values of 0.75 in each band, with a spurious counterpart rate of

\footnotetext{
${ }^{26}$ priOrSec $=0$ OR priOrSec $=$ frameSetId.

27 "Pnoise" is the probability that the detection is noise.
} 
20/1856, 39/1783, and 41/1763, respectively (see Figure 7). In total, 2117 XMM-Newton AO13 sources (74\% of the sample) have NIR counterparts from the VHS survey. We also check the "mergedClass" flag to test if a source is saturated ("mergedClass" $=-9$ ), but none of the matches are so afflicted.

Between UKIDSS and VHS, we find NIR counterparts for 2257 X-ray sources, or $79 \%$ of the sample. Of the NIR sources, 140 are found in UKIDSS, but not VHS. Of these, 34 were non-detections in VHS (i.e., no match between VHS and UKIDSS within a $2^{\prime \prime}$ search radius), while the remaining 106 were found in VHS but fell below our reliability thresholds for this catalog; we note that 77 of these VHS sources below the reliability cut had UKIDSS $Y$-band reliabilities above our $Y$-band critical threshold, while VHS is lacking this coverage. By presenting matches to both UKIDSS and VHS, the variability of the 1678 X-ray selected, NIR objects (1644 objects in common between UKIDSS and VHS and the 34 VHS dropouts) can be studied by the community.

\subsection{GALEX}

Similar to the UKIDSS matching, we used the cleaned GALEX catalog described in LaMassa et al. (2013a) to find counterparts to the XMM-Newton AO13 sources, matching to the near-ultraviolet (NUV) and far-ultraviolet (FUV) bands independently. This catalog represents data from the mediumimaging survey (MIS) in GALEX Release 7 (Morrissey et al. 2007). With a $R_{\text {crit }}$ value of 0.75 for both bands, we find 572 and 407 counterparts, with 12 and 5 spurious associations, in the NUV and FUV bands, respectively (see Figure 8). In total, $607 \mathrm{X}$-ray sources have ultraviolet counterparts, corresponding to $21 \%$ of the XMM-Newton AO13 sample.

\subsection{FIRST}

Due to the relatively low space density of the radio sources detected in the FIRST (Becker et al. 1995; White et al. 1997) survey, we used a nearest neighbor match to find counterparts to the X-ray sources, using the same search radius of $7^{\prime \prime}$ as employed in the MLE matching above. Similar to our previous Stripe $82 \mathrm{X}$ catalog release, we used the FIRST catalog published in 2012 which includes all sources detected between 1993 and 2011, which has a 0.75 mJy flux limit over the XMMNewton AO13 region (Becker et al. 2012). Since our previous paper, the final FIRST catalog has been published (Helfand et al. 2015) but we do not gain any additional sources when matching to this final catalog, both with the XMM-Newton AO13 data and archival Chandra and archival and AO10 XMM-Newton catalogs. Of the X-ray sample, 116 FIRST sources (4\% of the X-ray sample) are found within $7^{\prime \prime}$ of the XMM-Newton AO13 sources. When matching the FIRST catalog to the randomly shifted X-ray positions, eight spurious associations were found (see Figure 9).

\subsection{Herschel}

The Herschel Stripe 82 Survey (HerS) covers $79 \mathrm{deg}^{2}$ at 250, 350 , and $500 \mu \mathrm{m}$ to an average depth of 13.0, 12.9, and 14.8 mJy beam $^{-1}$ at $>3 \sigma$, surveyed with the Spectral and Photometric Imaging Receiver (SPIRE) instrument (Viero et al. 2014). The far-infrared emission from Herschel provides a clean tracer of host galaxy star-formation (Pier \&
Krolik 1992), making these data of particular importance to study the host galaxies of AGNs (Pier \& Krolik 1992; Efstathiou \& Rowan-Robinson 1995; Lutz et al. 2004; Fritz et al. 2006; Schweitzer et al. 2006; Netzer et al. 2007; Schartmann et al. 2008; Shao et al. 2010; Mullaney et al. 2011; Rosario et al. 2012; Magdis et al. 2013; Delvecchio et al. 2014). Indeed, the XMM-Newton AO13 survey was specifically designed to overlap existing Herschel coverage, since similar far-infrared data will not be available in the foreseeable future.

Similar to the matching to the FIRST catalog, we employed a nearest neighbor approach to find associations between the farinfrared Herschel sources and the X-ray objects. However, we shortened $r_{\text {search }}$ to $5^{\prime \prime}$ since our exercise of matching the Herschel catalog to the random X-ray positions reveals that most spurious associations occurred at distances between $5^{\prime \prime}$ and $7^{\prime \prime}$. We found 121 Herschel sources within $5^{\prime \prime}$ of the XMMNewton sources, corresponding to $4 \%$ of the sample, and 8 spurious associations when matching to the randomized X-ray positions (see Figure 10).

\subsection{XMM-Newton AO13 Multiwavelength Match Summary}

In total, we find counterparts to $93 \%$ of the XMM-Newton AO13 sources. However, we emphasize that we matched the $\mathrm{X}$-ray source list independently to each of the multi-wavelength catalogs and did not cross-correlate the counterparts. In a vast majority of the cases, these counterparts among the catalogs are the same source, though discrepancies exist. For guidance, we include a "cp_coord_flag" to note which sources have counterparts with consistent coordinates and which do not, using a search radius of $2^{\prime \prime}$ for SDSS, UKIDSS, VHS, and FIRST and $3^{\prime \prime}$ for WISE, GALEX, and Herschel due to the larger PSF and higher astrometric uncertainties in these latter catalogs compared with the former. When the coordinates are inconsistent within these search radii, the "cp_coord_flag" is set to one; otherwise it is set to null. For $89 \%$ of the X-ray sources with counterparts, their coordinates are consistent. We note, however, that above these search radii, consistent counterparts may exist and below these radii, there can still be discrepencies.

Finally, we highlight that the multi-wavelength magnitudes in the Stripe $82 \mathrm{X}$ catalogs may not be the most appropriate magnitude for every source and it is up to the user to determine whether different aperture photometry should be downloaded from the original catalog, using the identifying information presented in our catalogs to isolate the correct source, for the intended science goals.

A summary of the multi-wavelength columns and flags is presented in the appendix, as well as a discussion of updates made to the previously released Stripe $82 \mathrm{X}$ catalogs.

\section{DISCUSSION}

When considering the full Stripe $82 \mathrm{X}$ survey to date, including archival Chandra, archival XMM-Newton, XMMNewton AO10, and XMM-Newton AO13 data, we find multiwavelength counterparts to $88 \%$ of the X-ray sources. We are able to identify $\sim 30 \%$ of the Stripe $82 \mathrm{X}$ sample with spectroscopic objects. Of the sample, 67 objects are classified as stars while the remaining 1775 objects are extragalactic. We plot the $r$-band magnitude as a function of soft X-ray flux for all objects with optical counterparts in Figure 11, where we 


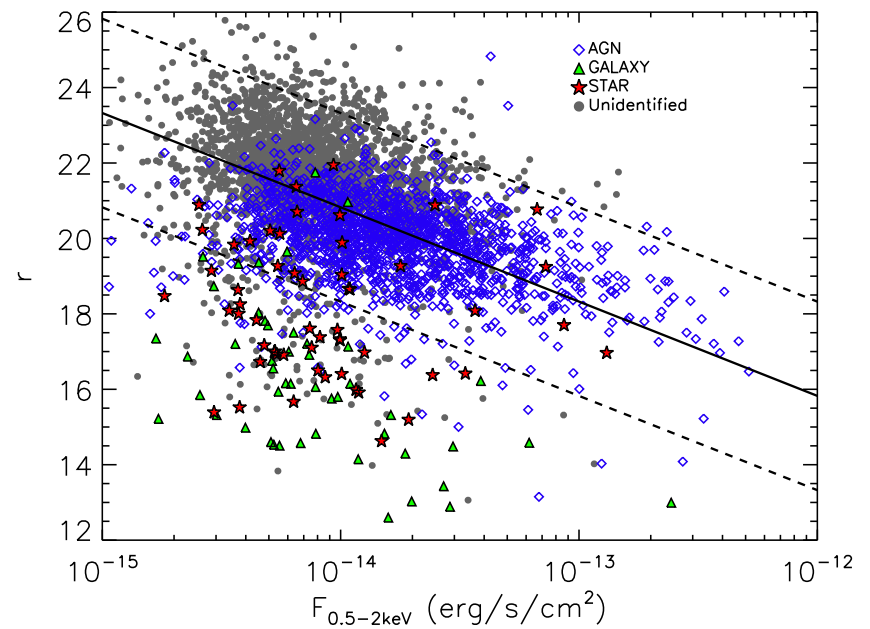

Figure 11. SDSS $r$-band magnitude as a function of observed $X$-ray flux in the $0.5-2 \mathrm{keV}$ band. The solid line defines the typical X-ray-to-optical flux ratio of AGNs (Brandt \& Hasinger 2005), while the dashed lines show the $\mathrm{X} / \mathrm{O}= \pm 1$ locus within which most AGNs lie (see Equation (3)). Stars are identified by their optical spectra while AGNs and galaxies are classified based on their observed $0.5-10 \mathrm{keV}$ luminosity, with $10^{42} \mathrm{erg} \mathrm{s}^{-1}$ being the dividing line.

note which objects are stars, X-ray AGNs, X-ray galaxies, and currently unidentified (i.e., they lack redshifts). Stars are classified on the basis of their optical spectra while here we use the observed, full-band X-ray luminosity to differentiate between X-ray AGNs $\left(L_{0.5-10 \mathrm{keV}}>10^{42} \mathrm{erg} \mathrm{s}^{-1}\right)$ and X-ray galaxies $\left(L_{0.5-10 \mathrm{keV}}<10^{42} \mathrm{erg} \mathrm{s}^{-1}\right)$, independent of their optical spectroscopic classification. For reference, we also include lines to mark typical AGNs X/O values (e.g., Brandt \& Hasinger 2005):

$$
\mathrm{X} / \mathrm{O}=\log \left(f_{x} / f_{\text {opt }}\right)=\log \left(f_{x}\right)+C+0.4 \times m_{\mathrm{r}},
$$

where $C$ is a constant based on the optical filter, which for the SDSS $r$-band, is 5.67 (see Green et al. 2004). Previous studies have found that AGNs generally fall within the $\mathrm{X} / 0=0 \pm 1$ locus (e.g., Schmidt et al. 1998; Alexander et al. 2001; Green et al. 2004; Brusa et al. 2007; Xue et al. 2011; Civano et al. 2012), as indicated by the dashed lines in Figure 11 . We find the same trend here, and note that extragalactic objects do not separate out from Galactic objects within this color space.

\subsection{Stars}

In Figure 12, we show how most X-ray emitting stars can be cleanly identified on the basis of their optical and infrared properties by comparing their $r-K$ and $r-W 1$ colors, as presented in LaMassa et al. (2015b). Here, we focus on the X-ray sources with SDSS, UKIDSS, and WISE counterparts that have $K$-band detections, $W 1$ detections ( $W 1$ SNR $>2$ ), and an $r$-band magnitude under 22.2 (the $95 \%$ completeness limit for the single-epoch SDSS imaging catalog) to avoid artificially inflating the colors to redder values. Additionally, we only retain the sources where the SDSS and UKIDSS coordinates are consistent within $2^{\prime \prime}$ and the SDSS and WISE coordinates agree within $3^{\prime \prime}$ to minimize spurious associations. In total, 1891 objects are shown in Figure 12, compared with the 4133 sources shown in the previous plot, which are sources detected in the $r$ and soft X-ray bands. Most of the stars follow a welldefined track in $r-K$ versus $r-W 1$ color space, aiding in the separation of Galactic and extragalactic candidates detected in

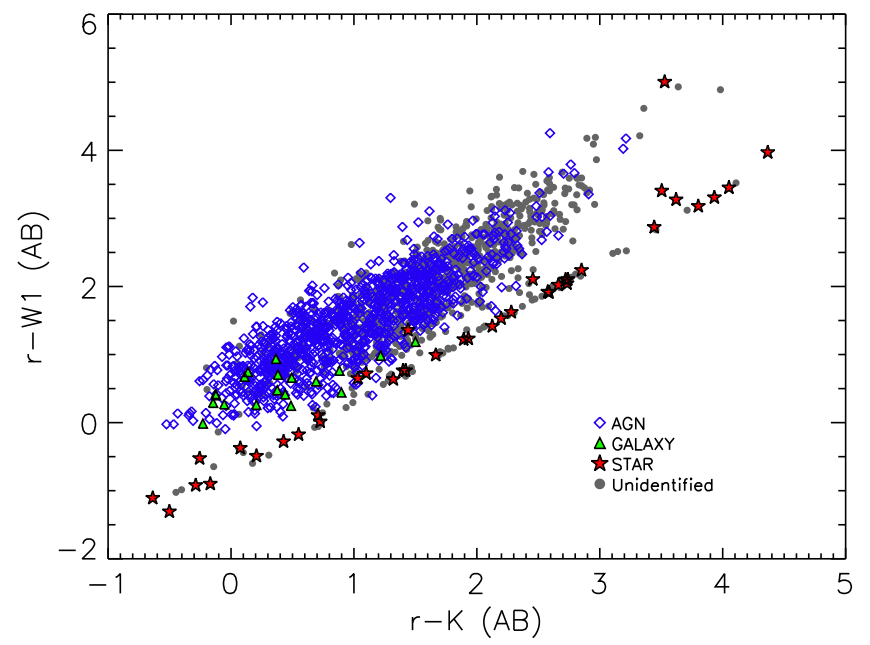

Figure 12. $r-W 1(\mathrm{AB})$ color as a function of $r-K(\mathrm{AB})$ color for the 1891 $\mathrm{X}$-ray sources with SDSS, UKIDSS, and WISE counterparts that have $K$-band and $W 1$ detections and UKIDSS (WISE) coordinates within $2^{\prime \prime}\left(3^{\prime \prime}\right)$ of the SDSS position. Many of the stars can be identified by the distinct track they occupy in this color space (LaMassa et al. 2015b).

X-ray surveys in the absence of supporting spectroscopic information.

\subsection{Extragalactic Objects}

In Figure 13 (left), we show the redshift distribution of the 1775 extragalactic sources with optical spectra: about half (875) are at $z>1$, with 301 at redshifts above 2 . We further break down the redshift distribution by classification, based on optical spectroscopy (see Section 4.2.1) and X-ray luminosity. In Figure 13, "broad-line" AGNs are sources optically classified as quasars due to broad emission lines in their spectra, "obscured AGNs" are sources optically classified as galaxies whose full-band observed X-ray luminosities exceed $10^{42} \mathrm{erg} \mathrm{s}^{-1}$, and "galaxies" are objects lacking broad-lines in their optical spectra whose X-ray luminosities are below $10^{42} \mathrm{erg} \mathrm{s}^{-1}$; we note, however, that this "galaxy" class can include Compton-thick AGNs $\left(N_{\mathrm{H}}>1.25 \times 10^{24} \mathrm{~cm}^{-2}\right)$ with very weak observed $\mathrm{X}$-ray emission due to heavy attenuation. Of the 1775 extragalactic sources in our sample, 19 are not classified in the spectroscopic databases we utilized and another 30 do not have significant detections in the full X-ray band.

The left-hand panel of Figure 13 demonstrates that nearly all the sources we have identified thus far at high redshifts (i.e., $z>1$ ) are broad-line AGNs, in part because unobscured quasars were preferentially selected as spectroscopic targets in the SDSS surveys. Most of the obscured AGNs live within the intermediate universe $(z \sim 0.5)$ while galaxies reside in the local universe $(z<0.25)$. We expect the percentage of obscured AGNs, i.e., those lacking broad emission lines, to increase as more objects are identified via spectroscopic and photometric redshifts.

In the right-hand panel of Figure 13, we show the observed full-band luminosity distribution of the X-ray AGNs, 1603 sources in total. The distribution peaks at relatively high luminosities ( $\sim 44.5$ dex $)$ due to the wide-area and shallow design of the survey. Most of the high luminosity AGNs are broad-line sources, though a handful of obscured AGNs do 

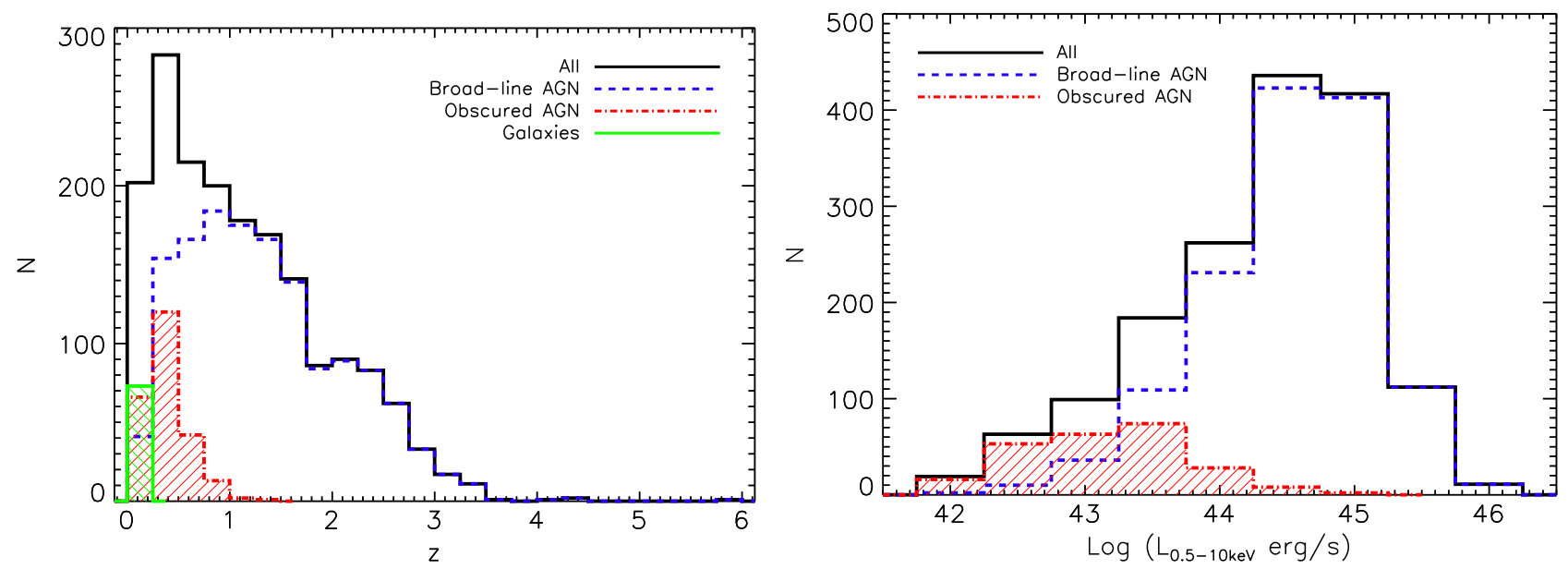

Figure 13. Left: spectroscopic redshift distribution of the 1775 extragalactic Stripe $82 \mathrm{X}$ sources, with different classes of objects highlighted. Half the sample is above a redshift of one, and contains predominantly broad-line AGNs at these distances. Nearly all obscured AGNs (i.e., sources optically classified as galaxies with but with full band X-ray luminosities above $10^{42} \mathrm{erg} \mathrm{s}^{-1}$ ) are at a redshift below 1, while the optical and X-ray galaxies are at $z<0.25$ (Compton-thick AGNs that have low observed X-ray flux due to heavy obscuration can be included in the "galaxy" bin). Right: observed full-band luminosity distribution for the 1603 spectroscopically confirmed X-ray AGNs (i.e., $\left.L_{0.5-10 \mathrm{keV}}>10^{42} \mathrm{erg} \mathrm{s}^{-1}\right)$, where the distribution peaks at high-luminosities $\left(44.25 \mathrm{dex}<\log \left(L_{0.5-10 \mathrm{keV}} \mathrm{erg} \mathrm{s}{ }^{-1}\right)<45.25 \mathrm{dex}\right)$. Highluminosity AGNs are predominantly broad-line sources while the lower-luminosity AGNs are mostly obscured. We note that these trends are for the $\sim 30 \%$ of the parent Stripe 82X sample that have spectroscopic redshifts and that with increased completeness and more sources identified via photometric redshifts, we expect to confirm more AGNs at all luminosities and redshifts, including at $z>2$ and $L_{0.5-10 \mathrm{keV}}>10^{45} \mathrm{erg} \mathrm{s}^{-1}$, and a higher percentage of obscured AGN.
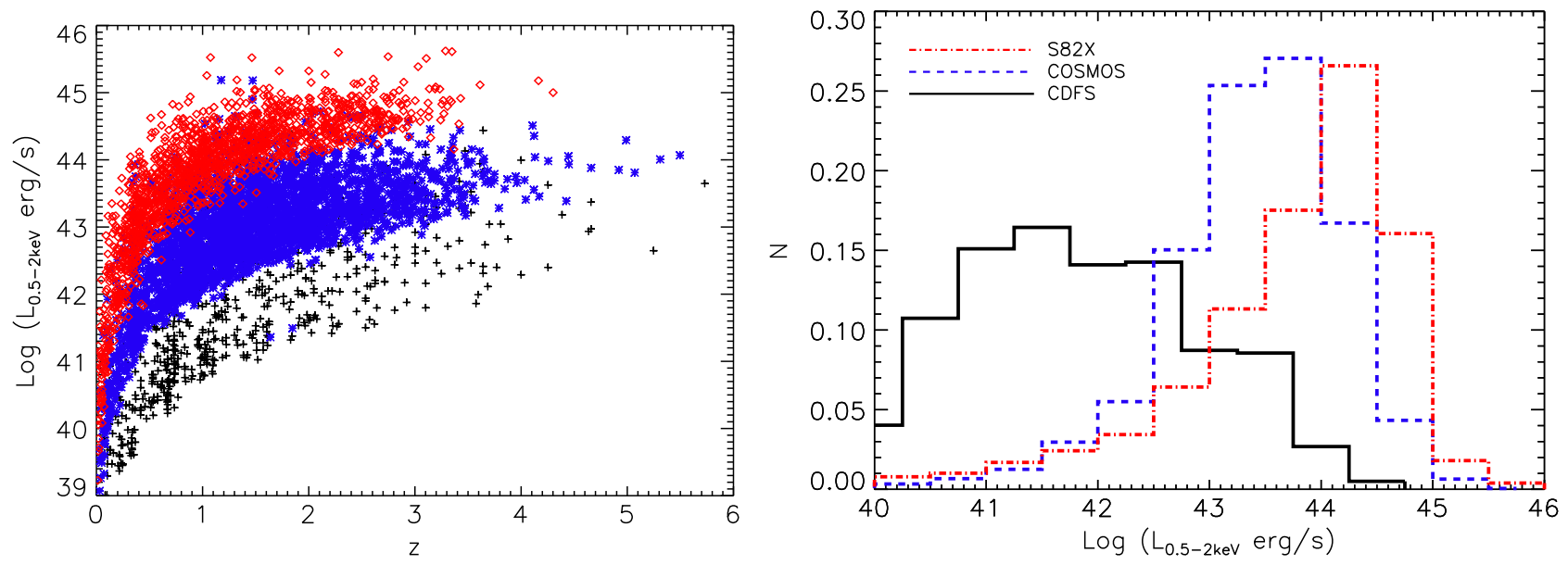

Figure 14. Left: $K$-corrected (rest-frame) soft-band (0.5-2 keV) luminosities as a function of redshift for the Stripe $82 \mathrm{X}$ (red diamonds), COSMOS-Legacy (blue asterisks; Civano et al. 2015; Marchesi et al. 2015), and CDFS (black crosses) sources. At every redshift, an increase in survey area preferentially identifies higherluminosity sources. Right: normalized distribution of $k$-corrected soft-band luminosities for Stripe 82X compared with COSMOS and CDFS: the wide-area coverage of Stripe $82 \mathrm{X}$ which probes a large effective volume of the universe, enables the rare, highest luminosity quasars to be uncovered, complementing the parameter space explored by small- to moderate-area surveys. In both plots, only the sources identified with redshifts are plotted, representing $30 \%$ of the Stripe $82 \mathrm{X}$ sample (which currently has only spectroscopic redshifts) and $91 \%$ and $96 \%$ of the CDFS and COSMOS-Legacy sample, respectively, where both spectroscopic and photometric redshifts are available.

reach moderately high X-ray luminosities $\left(\log \left(L_{0.5-10 \mathrm{kev}} / \mathrm{erg}\right.\right.$ $\left.\left.\mathrm{s}^{-1}\right)>43.75 \mathrm{dex}\right)$.

\subsection{The $L-z$ Plane Probed by Stripe $82 X$}

To put the Stripe $82 X$ sample in context with other surveys, we compare the luminosity-redshift plane with the small-area, deep CDFS survey $\left(0.13 \mathrm{deg}^{2}\right.$; Xue et al. 2011) and the moderate-area, moderate-depth COSMOS-Legacy survey (2.2 $\mathrm{deg}^{2}$; Civano et al. 2015; Marchesi et al. 2015). Here, we use soft-band $(0.5-2 \mathrm{keV})$ luminosities that have been $k$ corrected to the rest-frame, using $\Gamma=1.4$ for CDFS and COSMOS, while no $k$-correction was needed for Stripe $82 \mathrm{X}$ as the soft-band flux was estimated using $\Gamma=2$ and the $k$ correction scales as $(1+z)^{(\Gamma-2)}$. As Figure 14 (left) shows, as survey area increases and the effective flux limits of the surveys become shallower, the detected sources are preferentially at higher luminosity at every redshift. This is further illustrated in Figure 14 (right), which compares the normalized luminosity distribution of Stripe $82 \mathrm{X}$ with COSMOS and CDFS, highlighting the complementarity of the different survey strategies in preferentially identifying sources within different luminosity ranges (see, e.g., Hsu et al. 2014). Wide-area surveys which explore a large volume of the universe, like Stripe 82X, are necessary to discover rare objects that have a low space density, including the highest luminosity quasars.

One important caveat in Figure 14 is that we limit our comparison to sources with measured redshifts. For Stripe 82X, this represents the $30 \%$ of the sample that has spectroscopic redshifts while COSMOS and CDFS have spectroscopic and photometric redshifts, effectively identifying $\sim 96 \%$ and $\sim 91 \%$ 
of the parent samples, respectively. The photometric redshift catalog for Stripe $82 \mathrm{X}$ will be published in the coming months (T. Ananna et al 2015, in preparation), allowing us to identify the majority of the X-ray sources, increasing the number of sources at every redshift and luminosity.

\section{CONCLUSION}

We have presented the results from the most recent installment of the Stripe 82 X-ray survey, utilizing data awarded to our team in XMM-Newton cycle AO13. This program, amounting to $\sim 980 \mathrm{ks}$ of exposure time and spanning $15.6 \mathrm{deg}^{2}$, approximately doubled the previous X-ray coverage in the SDSS Stripe 82 Legacy field, with 2862 X-ray sources detected at $>5 \sigma$ level. The approximate flux limits of the AO13 portion of the Stripe $82 \mathrm{X}$ survey are $2.2 \times 10^{-15} \mathrm{erg} \mathrm{s}^{-1} \mathrm{~cm}^{-2}$, $1.3 \times 10^{-14} \mathrm{erg} \mathrm{s}^{-1} \mathrm{~cm}^{-2}$, and $6.7 \times 10^{-15} \mathrm{erg} \mathrm{s}^{-1} \mathrm{~cm}^{-2}$, in the soft $(0.5-2 \mathrm{keV})$, hard $(2-10 \mathrm{keV})$, and full $(0.5-10 \mathrm{keV})$ bands. From matching the X-ray source list to available multiwavelength catalogs, including SDSS (Ahn et al. 2012; Jiang et al. 2014), WISE (Wright et al. 2010; Mainzer et al. 2011), UKIDSS (Hewett et al. 2006; Casali et al. 2007; Lawrence et al. 2007; Warren et al. 2007), VHS (McMahon et al. 2013), GALEX (Morrissey et al. 2007), FIRST (Becker et al. 2012; Helfand et al. 2015), and Herschel (Viero et al. 2014), we identified reliable counterparts for $93 \%$ of the sample. About $29 \%$ of the X-ray sources are classified via spectroscopic redshifts.

Merging this data set with our previous releases of the Stripe 82X catalogs (LaMassa et al. 2013a, 2013b), the X-ray survey area in Stripe 82 covers $\sim 31.3 \mathrm{deg}^{2}$, with 6181 unique X-ray sources detected at $\geqslant 4.5 \sigma$ and $>5 \sigma$, for the Chandra and $X M M$-Newton components of the survey, respectively. We also updated the multi-wavelength matched X-ray catalogs for these earlier segments of the survey. In total, we find reliable multiwavelength counterparts for $88 \%$ of the full Stripe $82 \mathrm{X}$ survey to date, with a spectroscopic completeness of $30 \%$. We emphasize that we matched the X-ray source list to each multi-wavelength catalog independently and have not crosscorrelated the counterparts. However the counterparts largely agree among the catalogs, as discussed in the main text. Care must also be taken when studying the colors or spectral energy distributions of the X-ray sources using the magnitudes we present in these catalogs: it is up to the user to decide whether the aperture photometry in these catalogs is most suitable for a given source or if different aperture magnitudes should be retrieved from the main multi-wavelength catalogs, using the identifying information in the Stripe $82 \mathrm{X}$ catalog to select specific sources.

The large volume of the universe explored by the Stripe $82 \mathrm{X}$ survey enables the discovery of high-luminosity, high-redshift AGNs, a missing tier in the current X-ray census of supermassive black hole growth. We have several upcoming dedicated spectroscopic follow-up programs to increase the completeness of Stripe 82X, which in tandem with the photometric redshift catalog (T. Ananna et al. 2015, in preparation), will allow us to constrain how the most luminous $\mathrm{X}$-ray AGNs evolve over cosmic time. Furthermore, we are targeting obscured AGN candidates which have optical and infrared clues that they may be the transitional link in the merger-induced black hole growth/galaxy evolution paradigm (see, e.g., Glikman et al. 2013; Brusa et al. 2015); a handful of such luminous obscured AGNs at $z>1$ have already been discovered (S. M. LaMassa et al. 2015, in preparation), with many more candidates yet to be explored in this data set. Additionally, Stripe $82 \mathrm{X}$ will provide insight into AGN host galaxies via spectral energy distribution analysis, AGN variability, the dark matter halos hosting high-luminosity quasars at high-redshift via clustering analysis, the X-ray properties of galaxy clusters, and the opportunity to discover exotic sources. For instance, in the previous release of the Stripe $82 \mathrm{X}$ catalog, we discovered the first "changing-look" quasar (LaMassa et al. 2015a), an AGN which transitioned from a broad-line (Type 1) object to a mostly narrow-line (Type 1.9) object within a 10 year period (see also Merloni et al. 2015). We expect that Stripe $82 \mathrm{X}$ will have applications beyond those listed here, and can be particularly helpful in informing best-effort practices for AGN identification and follow-up in upcoming wide-area surveys, including $e$ ROSITA which will be launched in 2017 (Merloni et al. 2012; Predehl et al. 2014). Finally, we note that increasing the X-ray area to $100 \mathrm{deg}^{2}$ will open a new window into black hole growth at $z>3$ and luminosities greater than $10^{45} \mathrm{erg} \mathrm{s}^{-1}$, which is only beginning to be explored from an X-ray perspective (e.g., Georgakakis et al. 2015). The existing ancillary data will allow these objects to be readily characterized, allowing this population to be fully understood.

We thank the anonymous referee for feedback that helped improve the manuscript. S.M.L. acknowledges support from grant number NNX15AJ40G. C.M.U. gratefully acknowledges support from Yale University. C.M.U. and S.K. would like to thank the Kavli Institute for Theoretical Physics (Santa Barbara) for their hospitality and support; the KITP is supported by NSF grant No. NSF PHY11-25915. M.B. acknowledges support from the FPY Career Integration Grant "eEASY" (CIG 321913). Support for the work of E.T. was provided by the Center of Excellence in Astrophysics and Associated Technologies (PFB 06), by the FONDECYT grant 1120061 and the Anillo project ACT1101. K.S. gratefully acknowledges support from Swiss National Science Foundation Grant PP00P2_138979/1.

Funding for the SDSS and SDSS-II has been provided by the Alfred P. Sloan Foundation, the Participating Institutions, the National Science Foundation, the U.S. Department of Energy, the National Aeronautics and Space Administration, the Japanese Monbukagakusho, the Max Planck Society, and the Higher Education Funding Council for England. The SDSS Web Site is http://www.sdss.org/.

The SDSS is managed by the Astrophysical Research Consortium for the Participating Institutions. The Participating Institutions are the American Museum of Natural History, Astrophysical Institute Potsdam, University of Basel, University of Cambridge, Case Western Reserve University, University of Chicago, Drexel University, Fermilab, the Institute for Advanced Study, the Japan Participation Group, Johns Hopkins University, the Joint Institute for Nuclear Astrophysics, the Kavli Institute for Particle Astrophysics and Cosmology, the Korean Scientist Group, the Chinese Academy of Sciences (LAMOST), Los Alamos National Laboratory, the Max-Planck-Institute for Astronomy (MPIA), the Max-PlanckInstitute for Astrophysics (MPA), New Mexico State University, Ohio State University, University of Pittsburgh, University of Portsmouth, Princeton University, the United States Naval Observatory, and the University of Washington. 
Table 5

Common Columns among All X-Ray Catalogs: X-Ray Information

\begin{tabular}{|c|c|}
\hline Column & Description \\
\hline ObsID & Chandra or XMM-Newton observation identification number. \\
\hline R.A. & X-ray R.A. (J2000). \\
\hline decl. & X-ray decl. (J2000). \\
\hline RADec_err & Positional error on the X-ray coordinates in arcseconds. \\
\hline Dist_NN & Distance to the nearest X-ray source in the catalog in arcseconds. \\
\hline $\begin{array}{l}\text { Removed_LogN_LogS (Chandra and XMM-Newton archival } \\
\text { and AO10 catalogs only) }\end{array}$ & $\begin{array}{l}\text { Flag set to "yes" if removed from the } \log N-\log S \text { relations presented here. The removed } \\
\text { objects are targeted sources of observations, and in the case of the Chandra catalog, objects } \\
\text { that overlap XMM-Newton observations in the field. }\end{array}$ \\
\hline Soft_Flux & $\begin{array}{l}\text { Observed X-ray flux in the soft }(0.5-2 \mathrm{keV}) \text { band }\left(\mathrm{erg} \mathrm{s}^{-1} \mathrm{~cm}^{-2}\right) \text {. For the Chandra sources, only } \\
\text { significant }(\geqslant 4.5 \sigma) \text { fluxes are reported (see the text for details) while all fluxes are reported for } \\
\text { the XMM-Newton sources, where users should refer to the "soft_detml" entry to determine } \\
\text { appropriate level of source significance suitable for analysis. Fluxes are converted from count } \\
\text { rate assuming a power-law spectrum where } \Gamma=2.0 \text {. }\end{array}$ \\
\hline Soft_Counts & Net counts in the soft $(0.5-2 \mathrm{keV})$ band. \\
\hline Hard_Flux & $\begin{array}{l}\text { Observed X-ray flux in the hard band, which corresponds to the } 2-7 \mathrm{keV} \text { range for Chandra and } \\
2-10 \mathrm{keV} \text { range for } X M M \text {-Newton (erg s} \mathrm{em}^{-1} \mathrm{~cm}^{-2} \text { ). For the Chandra sources, only significant } \\
(\geqslant 4.5 \sigma) \text { fluxes are reported (see the text for details) while all fluxes are reported for the } X M M- \\
\text { Newton sources, where users should refer to the "hard_detml" entry to determine the appro- } \\
\text { priate level of source significance suitable for analysis. Fluxes are converted from count rate } \\
\text { assuming a power-law spectrum where } \Gamma=1.7 \text {. }\end{array}$ \\
\hline Hard_Counts & $\begin{array}{l}\text { Net counts in the hard band }(2-7 \mathrm{keV} \text { and } 2-10 \mathrm{keV} \text { for Chandra and XMM-Newton, } \\
\text { respectively). }\end{array}$ \\
\hline Full_Flux & $\begin{array}{l}\text { Observed X-ray flux in the full band, which corresponds to the } 0.5-7 \mathrm{keV} \text { range for Chandra and } \\
0.5-10 \mathrm{keV} \text { range for } X M M \text {-Newton (erg s }{ }^{-1} \mathrm{~cm}^{-2} \text { ). For the Chandra sources, only significant } \\
(\geqslant 4.5 \sigma) \text { fluxes are reported (see the text for details) while all fluxes are reported for the } X M M \text { - } \\
\text { Newton sources, where users should refer to the "full_detml" entry to determine appropriate } \\
\text { level of source significance suitable for analysis. Fluxes are converted from count rate } \\
\text { assuming a power-law spectrum where } \Gamma=1.7 \text {. }\end{array}$ \\
\hline Full_Counts & $\begin{array}{l}\text { Net counts in the full band ( } 0.5-7 \mathrm{keV} \text { and } 0.5-10 \mathrm{keV} \text { for Chandra and XMM-Newton, } \\
\text { respectively). }\end{array}$ \\
\hline Lum_Soft & Log of the observed luminosity in the soft $(0.5-2 \mathrm{keV})$ band in units of erg $\mathrm{s}^{-1}$. \\
\hline Lum_Hard & $\begin{array}{l}\text { Log of the observed luminosity in the hard band ( } 2-7 \mathrm{keV} \text { and } 2-10 \mathrm{keV} \text { for Chandra and XMM- } \\
\text { Newton, respectively), in units of erg s }{ }^{-1} \text {. }\end{array}$ \\
\hline Lum_Full & $\begin{array}{l}\text { Log of the observed luminosity in the full band }(0.5-7 \mathrm{keV} \text { and } 0.5-10 \mathrm{keV} \text { for Chandra and } \\
\text { XMM-Newton respectively), in units of erg s }{ }^{-1} \text {. }\end{array}$ \\
\hline
\end{tabular}

Funding for SDSS-III has been provided by the Alfred P. Sloan Foundation, the Participating Institutions, the National Science Foundation, and the U.S. Department of Energy Office of Science. The SDSS-III web site is http://www.sdss3.org/.

SDSS-III is managed by the Astrophysical Research Consortium for the Participating Institutions of the SDSS-III Collaboration including the University of Arizona, the Brazilian Participation Group, Brookhaven National Laboratory, Carnegie Mellon University, University of Florida, the French Participation Group, the German Participation Group, Harvard University, the Instituto de Astrofisica de Canarias, the Michigan State/Notre Dame/JINA Participation Group, Johns Hopkins University, Lawrence Berkeley National Laboratory, Max Planck Institute for Astrophysics, Max Planck Institute for Extraterrestrial Physics, New Mexico State University, New York University, Ohio State University, Pennsylvania State University, University of Portsmouth, Princeton University, the Spanish Participation Group, University of Tokyo, University of Utah, Vanderbilt University, University of Virginia, University of Washington, and Yale University.

This publication makes use of data products from the Widefield Infrared Survey Explorer, which is a joint project of the University of California, Los Angeles, and the Jet Propulsion Laboratory/California Institute of Technology, funded by the National Aeronautics and Space Administration.

\section{APPENDIX A UPDATES TO PREVIOUSLY RELEASED STRIPE 82X CATALOGS}

The archival Chandra, archival XMM-Newton plus AO10, and XMM-Newton AO13 sources are available in FITS format catalogs in the electronic edition. Updates to previous versions of these catalogs are provided below.

As mentioned in Section 4, the MLE matching between the archival XMM-Newton and AO10 source lists and ancillary catalogs was updated to include a 1 " systematic error added in quadrature to the emldetect reported positional error. Since the Chandra Source Catalog has external astrometric corrections applied (Rots \& Budavári 2011), a systematic positional error did not need to be added in by hand for this source list. Additional updates to the previously published catalogs are listed below, where Table 4 summarizes the multi-wavelength catalog matching for all components of the Stripe 82X survey. Tables 5-8 describe the common and unique FITS headers associated with each catalog.

\section{A.1. X-Ray Catalogs}

In the X-ray source lists for both the XMM-Newton and Chandra catalogs, we now include columns for net counts detected in the soft, hard, and full bands. We also updated the 
Table 6

Common Columns Among all Catalogs: Multi-wavelength Information

\begin{tabular}{|c|c|}
\hline Column & Description \\
\hline SDSS_rej & $\begin{array}{l}\text { Flag set to "yes" if SDSS counterpart is found which exceeds the reliability threshold, but the photometry was rejected for failing quality control } \\
\text { checks. }\end{array}$ \\
\hline SDSS_OBJID & SDSS object identification number of SDSS counterpart to X-ray source. \\
\hline SDSS_RA & SDSS R.A. of counterpart (J2000). \\
\hline SDSS_Dec & SDSS decl. of counterpart (J2000). \\
\hline SDSS_Rel & MLE reliability value of SDSS counterpart; highest of the $u, g, r, i$, and $z$ reliability values. \\
\hline SDSS_Dist & Distance between X-ray source and SDSS counterpart in arcseconds. \\
\hline SDSS_Coadd & $\begin{array}{l}\text { Flag set to "yes" if SDSS counterpart is found from the Jiang et al. (2014) coadded catalog. Otherwise, the SDSS counterpart was identified in the } \\
\text { single-epoch DR9 imaging catalog. }\end{array}$ \\
\hline u_mag & $\begin{array}{l}\text { SDSS } u \text {-band magnitude. For the single-epoch matches, this value represents the SDSS pipeline reported ModelMag, while the sources from the } \\
\text { coadded SDSS catalog have Mag_Auto values calculated via SExtractor (Bertin \& Arnouts 1996) as reported in the Jiang et al. (2014) catalogs. }\end{array}$ \\
\hline u_err & $\begin{array}{l}\text { SDSS } u \text {-band magnitude error. For the single-epoch matches, this value represents the SDSS pipeline reported ModelMagErr, while the sources } \\
\text { from the coadded SDSS catalog have MagErr_Auto values calculated via SExtractor (Bertin \& Arnouts 1996) as reported in the Jiang et al. } \\
\text { (2014) catalogs. }\end{array}$ \\
\hline g_mag & SDSS $g$-band magnitude. See $u \_$mag for details. \\
\hline g_err & SDSS $g$-band magnitude error. See $u \_$err for details. \\
\hline r_mag & SDSS $r$-band magnitude. See $u \_$mag for details. \\
\hline r_err & SDSS $r$-band magnitude error. See $u$ _err for details. \\
\hline i_mag & SDSS $i$-band magnitude. See $u \_m a g$ for details. \\
\hline i_err & SDSS $i$-band magnitude error. See $u_{-}$err for details. \\
\hline z_mag & SDSS $z$-band magnitude. See $u \_$mag for details. \\
\hline z_err & SDSS $z$-band magnitude error. See $u \_$err for details. \\
\hline Specobjid & SDSS spectroscopic identification number. \\
\hline Class & $\begin{array}{l}\text { Optical spectroscopic class as indicated by pipeline processing (for spectra downloaded from existing databases) or determined by us through } \\
\text { visual inspection for sources targeted in our follow-up campaigns. QSO—broad emission lines in spectra; GALAXY—narrow emission lines } \\
\text { and/or absorption lines only; STAR—stellar spectrum. }\end{array}$ \\
\hline Redshift & Spectroscopic redshift. \\
\hline Z_src & 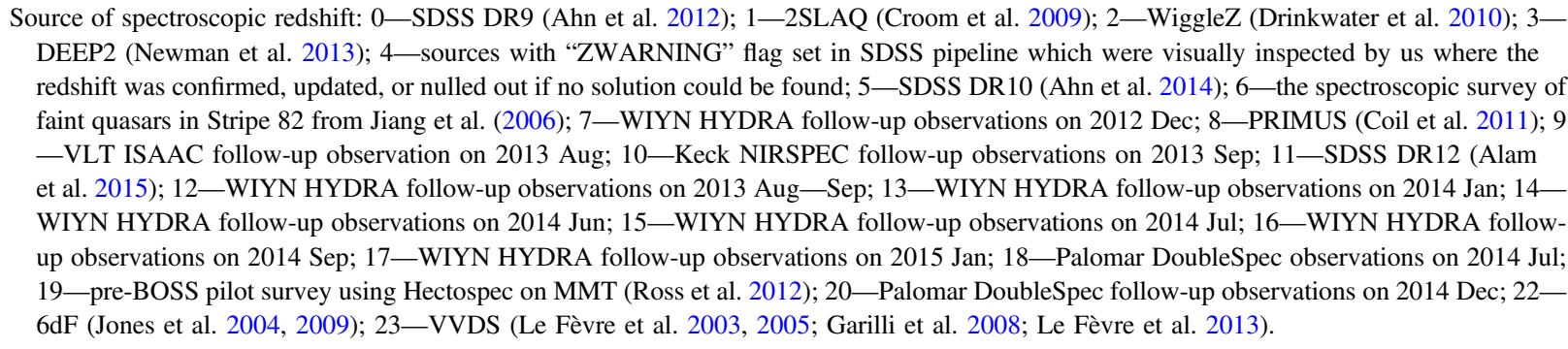 \\
\hline WISE_Name & WISE name. \\
\hline WISE_RA & R.A. of WISE counterpart (J2000). \\
\hline WISE_Dec & decl. of WISE counterpart (J2000). \\
\hline WISE_sigra & Uncertainty of WISE R.A. (arcsecond). \\
\hline WISE_sigdec & Uncertainty of WISE decl. (arcsecond). \\
\hline WISE_Rel & MLE reliability value of WISE counterpart. \\
\hline WISE_Dist & Distance between WISE counterpart and X-ray source (arcsecond). \\
\hline W1 & W1 magnitude (Vega). \\
\hline W1sig & $W 1$ error. \\
\hline W1SNR & $W 1$ signal-to-noise ratio. Magnitudes with $\mathrm{SNR}<2$ are upper limits. \\
\hline W2 & $W 2$ magnitude (Vega). \\
\hline W2sig & $W 2$ error. \\
\hline W2SNR & $W 2$ signal-to-noise ratio. Magnitudes with $\mathrm{SNR}<2$ are upper limits. \\
\hline W3 & W3 magnitude (Vega). \\
\hline W3sig & $W 3$ error. \\
\hline W3SNR & W3 signal-to-noise ratio. Magnitudes with SNR $<2$ are upper limits. \\
\hline W4 & W4 magnitude (Vega). \\
\hline W4sig & $W 4$ error. \\
\hline W4SNR & W4 signal-to-noise ratio. Magnitudes with $\mathrm{SNR}<2$ are upper limits. \\
\hline WISE_ext & Flag set to "yes" if the WISE source is extended. \\
\hline WISE_rej & $\begin{array}{l}\text { Flag set to "yes" if WISE counterpart is identified via MLE matching but the source is rejected due to failing photometry control checks in every } \\
\text { WISE band. }\end{array}$ \\
\hline UKIDSS_ID & Identification number of UKIDSS counterpart. \\
\hline UKIDSS_RA & R.A. of UKIDSS counterpart (J2000). \\
\hline UKIDSS_Dec & decl. of UKIDSS counterpart (J2000). \\
\hline UKIDSS_Rel & MLE reliability value of UKIDSS counterpart; highest of the $Y, J, H$, and $K$ reliability values. \\
\hline
\end{tabular}


Table 6

(Continued)

\begin{tabular}{|c|c|}
\hline Column & Description \\
\hline UKIDSS_Dist & Distance between UKIDSS counterpart and X-ray source (arcsecond). \\
\hline UKIDSS_Ymag & UKIDSS $Y$ magnitude (Vega). \\
\hline UKIDSS_Ysig & UKIDSS $Y$ magnitude error (Vega). \\
\hline UKIDSS_Jmag & UKIDSS $J$ magnitude (Vega). \\
\hline UKIDSS_Jsig & UKIDSS $J$ magnitude error (Vega). \\
\hline UKIDSS_Hmag & UKIDSS $H$ magnitude (Vega). \\
\hline UKIDSS_Hsig & UKIDSS $H$ magnitude error (Vega). \\
\hline UKIDSS_Kmag & UKIDSS $K$ magnitude (Vega). \\
\hline UKIDSS_Ksig & UKIDSS $K$ magnitude error (Vega). \\
\hline UKIDSS_rej & flag set to "yes" if UKIDSS counterpart is found via MLE matching but source is rejected due to failing quality control checks. \\
\hline VHS_ID & Identification number of VHS counterpart. \\
\hline VHS_RA & R.A. of VHS counterpart (J2000). \\
\hline VHS_Dec & decl. of VHS counterpart (J2000). \\
\hline VHS_Rel & MLE reliability value of VHS counterpart; highest of the $J, H$, and $K$ reliability values. \\
\hline VHS_Dist & Distance between VHS counterpart and X-ray source (arcsecond). \\
\hline VHS_Jmag & VHS $J$ magnitude (Vega). \\
\hline VHS_Jsig & VHS $J$ magnitude error (Vega). \\
\hline VHS_Hmag & VHS $H$ magnitude (Vega). \\
\hline VHS_Hsig & VHS $H$ magnitude error (Vega). \\
\hline VHS_Kmag & VHS $K$ magnitude (Vega). \\
\hline VHS_Ksig & VHS $K$ magnitude error (Vega). \\
\hline VHS_rej & flag set to "yes" if VHS counterpart is found via MLE matching but source is rejected due to failing quality control checks. \\
\hline GALEX_RA & R.A. of GALEX counterpart (J2000). \\
\hline GALEC_Dec & decl. of GALEX counterpart (J2000). \\
\hline NUV_poserr & Positional error on the NUV GALEX position (arcsecond). \\
\hline FUV_poserr & Positional error on the FUV GALEX position (arcsecond). \\
\hline GALEX_Rel & MLE reliability value of the GALEX counterpart; higher of the FUV and NUV reliability values. \\
\hline GALEX_Dist & Distance between GALEX counterpart and X-ray source (arcsecond). \\
\hline NUV_Mag & NUV magnitude $(\mathrm{AB})$ \\
\hline NUV_MagErr & Error on NUV magnitude. \\
\hline FUV_Mag & FUV magnitude (AB). \\
\hline FUV_MagErr & Error on FUV magnitude. \\
\hline FIRST_Name & Name of FIRST counterpart. \\
\hline FIRST_RA & R.A. of FIRST counterpart (J2000). \\
\hline FIRST_Dec & decl. of FIRST counterpart (J2000). \\
\hline FIRST_Flux & Integrated flux density at $1.4 \mathrm{GHz}(\mathrm{mJy})$ \\
\hline FIRST_Err & Error on the flux density, calculated by multiplying the integrated flux density by the ratio of the RMS to the peak flux (mJy). \\
\hline HERS_RA & R.A. of Herschel counterpart from HeRS survey (J2000; Viero et al. 2014). \\
\hline HERS_Dec & decl. of Herschel counterpart from HeRS survey (J2000; Viero et al. 2014). \\
\hline F 250 & Flux density at $250 \mu \mathrm{m}(\mathrm{mJy})$ \\
\hline F250_Err & $1 \sigma$ flux density error at $250 \mu \mathrm{m}$ (mJy). \\
\hline F350 & Flux density at $350 \mu \mathrm{m}(\mathrm{mJy})$ \\
\hline F350_Err & $1 \sigma$ flux density error at $350 \mu \mathrm{m}$ (mJy). \\
\hline F500 & Flux density at $500 \mu \mathrm{m}(\mathrm{mJy})$ \\
\hline F500_err & $1 \sigma$ flux error at $500 \mu \mathrm{m}(\mathrm{mJy})$ \\
\hline cp_coord_flag & $\begin{array}{l}\text { Set to } 0 \text { if multi-wavelength counterpart coordinates are consistent within } 2^{\prime \prime} \text { (SDSS, UKIDSS, VHS, FIRST) or } 3^{\prime \prime} \text { (WISE, GALEX, Herschel); } \\
\text { otherwise flag is set to } 1 \text {. }\end{array}$ \\
\hline
\end{tabular}

XMM-Newton catalog to include an "ext_flag" whereas the previous version had the fluxes in the band that were fit as extended by emldetect set to zero; these fluxes now reflect the values reported by emldetect. Additionally, in the previous $X M M$-Newton catalog, we set to null any flux that was not detected at the det_ml $\geqslant 15$ level. Here, we report the fluxes along with their corresponding det_ml value for the user to decide which flux significance is most appropriate for their purposes. For fluxes from the Chandra source list, we determined the $4.5 \sigma$ significance by comparing the catalog with the sensitivity maps (see LaMassa et al. 2013b), nulling out any fluxes which were below this significance threshold; we refer the user to the Chandra Source Catalog (Evans et al. 2010) for flux measurements at lower significance.
The "removed_logn_logs" flags have been updated in both catalogs to indicate which sources were excluded from the $\log N-\log S$ generation in this work. These discarded sources represent those that are in overlapping observations that were excluded from the area-flux curve or that were targeted sources in archival observations.

Finally, in the previous catalog releases, we noted whether a Chandra source was found in the XMM-Newton catalog and vice versa, using the matching algorithm discussed in the main text to find matches between the XMM-Newton source lists generated via emldetect. We added a column to note whether a source is also detected in the XMM-Newton AO13 catalog, as well as the unique identifying information for the matched source (i.e., the Chandra MSID if the "in_chandra" flag is set 
Table 7

Additional Columns in Chandra Catalog

\begin{tabular}{|c|c|}
\hline Column & Description \\
\hline MSID & Chandra Source Catalog unique identification number. \\
\hline Soft_Flux_Error_High & Higher bound on $0.5-2 \mathrm{keV}$ flux ( $\mathrm{erg} \mathrm{s}^{-1} \mathrm{~cm}^{-2}$ ). If soft flux is zero, this represents the upper limit. \\
\hline Soft_Flux_Error_Low & Lower bound on $0.5-2 \mathrm{keV}$ flux $\left(\mathrm{erg} \mathrm{s}^{-1} \mathrm{~cm}^{-2}\right)$. \\
\hline Hard_Flux_Error_High & Higher bound on $2-7 \mathrm{keV}$ flux ( $\mathrm{erg} \mathrm{s}^{-1} \mathrm{~cm}^{-2}$ ). If hard flux is zero, this represents the upper limit. \\
\hline Hard_Flux_Error_Low & Lower bound on $2-7 \mathrm{keV}$ flux $\left(\mathrm{erg} \mathrm{s}^{-1} \mathrm{~cm}^{-2}\right)$. \\
\hline Full_Flux_Error_High & Higher bound on $0.5-7 \mathrm{keV}$ flux ( $\mathrm{erg} \mathrm{s}^{-1} \mathrm{~cm}^{-2}$ ). If full flux is zero, this represents the upper limit. \\
\hline Full_Flux_Error_Low & Lower bound on $0.5-7 \mathrm{keV}$ flux $\left(\mathrm{erg} \mathrm{s}^{-1} \mathrm{~cm}^{-2}\right)$. \\
\hline In_XMM & $\begin{array}{l}\text { Flag set to "yes" if source is detected in the archival and AO10 XMM-Newton catalog. The rec_no of this associated XMM-Newton source } \\
\text { is also given. }\end{array}$ \\
\hline In_XMM_AO13 & Flag set to "yes" if source is detected in the AO13 XMM-Newton catalog. The rec_no of this associated XMM-Newton source is also given. \\
\hline XMM_archive_cp_flag & $\begin{array}{l}\text { Flag to indicate that a multi-wavelength counterpart is promoted into this catalog: the multi-wavelength association did not meet the MLE } \\
\text { reliability threshold when matching to the Chandra catalog, but did meet this requirement for the same X-ray source in the XMM- } \\
\text { Newton archival catalog. The number indicates from which catalog the promoted match is found: } 1 \text {-SDSS counterpart found but } \\
\text { photometry rejected for failing quality control checks; 2-SDSS; 3-redshift; 4-WISE counterpart found but rejected for failing quality } \\
\text { control checks; 5-WISE; 6-UKIDSS; 7-VHS; 8-GALEX. }\end{array}$ \\
\hline XMM_ao13_cp_flag & $\begin{array}{l}\text { Similar to the "XMM_archive_cp_flag," but for matches from the archival XMM-Newton AO13 catalog. See "XMM_archive_cp_flag" for } \\
\text { more information. }\end{array}$ \\
\hline
\end{tabular}

Table 8

Additional Columns in XMM-Newton Catalog

\begin{tabular}{|c|c|}
\hline Column & Description \\
\hline Rec_No & Unique identifying number for $\mathrm{X}$-ray source. \\
\hline Ext_Flag & $\begin{array}{l}\text { Flag to indicate whether source was extended in one or more bands while being point-like in another band: } 1 \text { - } \\
\text { extended in the soft band, } 2 \text { - extended in the full band, } 3 \text { - extended in the hard band, } 4 \text {-extended in the } \\
\text { soft and full bands, } 5 \text { - extended in the soft and hard bands, } 6 \text {-extended in the hard and full bands. If } 0 \text {, } \\
\text { then the source is point-like in all bands. }\end{array}$ \\
\hline In_XMM (in AO13 catalog) & $\begin{array}{l}\text { Flag set to "yes" if source is detected in the archival and AO10 XMM-Newton catalog. The rec_no of this } \\
\text { associated XMM-Newton source is also given. }\end{array}$ \\
\hline In_XMM_AO13 (in archival and AO10 catalog) & $\begin{array}{l}\text { Flag set to "yes" if source is detected in the XMM-Newton AO13 catalog. The rec_no of this associated XMM- } \\
\text { Newton source is also given. }\end{array}$ \\
\hline In_Chandra & $\begin{array}{l}\text { Flag set to "yes" if source is detected in the Chandra catalog. The MSID of this associated Chandra source is } \\
\text { also given. }\end{array}$ \\
\hline Soft_Flux_Err & Error on the $0.5-2 \mathrm{keV}$ flux $\left(\mathrm{erg} \mathrm{s}^{-1} \mathrm{~cm}^{-2}\right)$ \\
\hline Soft_detml & $\begin{array}{l}\text { Significance of the detection in the } 0.5-2 \mathrm{keV} \text { band, where } \text { det_}_{-} m l=-\ln P_{\text {random. }} \text {. Users are cautioned to } \\
\text { determine the flux significance necessary for their science goals before utilizing the reported flux. For } \\
\text { reference, we only include objects where } \text { det }_{-} m l \geqslant 15 \text { in the } \log N-\log S \text { relationship, and only report the } \\
\text { luminosities for objects above this threshold. }\end{array}$ \\
\hline Hard_Flux_Err & Error on the $2-10 \mathrm{keV}$ flux $\left(\mathrm{erg} \mathrm{s}^{-1} \mathrm{~cm}^{-2}\right)$ \\
\hline Hard_detml & Significance of the detection in the $2-10 \mathrm{keV}$ band. See "soft_detml" for more information. \\
\hline Full_Flux_Err & Error on the $0.5-10 \mathrm{keV}$ flux $\left(\mathrm{erg} \mathrm{s}^{-1} \mathrm{~cm}^{-2}\right)$ \\
\hline Full_detml & Significance of the detection in the $0.5-10 \mathrm{keV}$ band. See "soft_detml" for more information. \\
\hline $\begin{array}{l}\text { XMM_ao13_cp_flag (in archival and } \mathrm{AO} 10 \\
\text { catalog) }\end{array}$ & $\begin{array}{l}\text { Flag to indicate that a multi-wavelength counterpart is promoted into this catalog: the multi-wavelength } \\
\text { association did not meet the MLE reliability threshold when matching to the AO13 catalog, but did meet this } \\
\text { requirement for the same X-ray source in the XMM-Newton archival catalog. The number indicates from } \\
\text { which catalog the promoted match is found: } 1 \text {-SDSS counterpart found but photometry rejected for failing } \\
\text { quality control checks;2-SDSS; 3-redshift; 4-WISE counterpart found but rejected for failing quality } \\
\text { control checks; 5-WISE; 6-UKIDSS; 7-VHS; 8-GALEX. }\end{array}$ \\
\hline XMM_archive_cp_flag (in AO13 catalog) & $\begin{array}{l}\text { Same as the "XMM_ao13_cp_flag," but for promoted matches into the AO13 catalog from the archival and } \\
\text { AO10 catalog. }\end{array}$ \\
\hline Ch_cp_flag & $\begin{array}{l}\text { Similar to the "XMM_ao13_cp_flag," but for matches from the archival Chandra catalog. See "XMM_arch- } \\
\text { ive_cp_flag" for more information. }\end{array}$ \\
\hline
\end{tabular}

to "yes" and the XMM-Newton record number if the "in_xmm" or "in_xmm_ao13" flag is set to "yes").

\section{A.2. Coadded SDSS Catalog}

We followed the same procedure detailed above when matching the previously released X-ray catalogs to the coadded

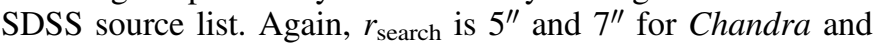
XMM-Newton, respectively (Brusa et al. 2010; Civano et al.
2012; LaMassa et al. 2013a). The $R_{\text {crit }}$ values when matching to the Chandra source list are as follows: $u-0.7, g-0.9, r-$ $0.85, i-0.85$, and $z-0.8$, with the fraction of random to true matches above this threshold being 14/572, 16/543, 19/601, $18 / 601$, and $13 / 816$, respectively. For XMM-Newton, we impose $R_{\text {crit }}$ values of $0.85,0.9,0.9,0.9,0.85$ in the $u, g, r, i$, and $z$ bands, respectively, with spurious fractions of 29/1290, 39/1326, 60/1283, 54/1228, and 49/1420. We retain the coadded match if there is not a counterpart found in the single- 
epoch imaging. By matching to the coadded catalog, we gain additional optical counterparts to 139 of the Chandra sources and 250 of the XMM-Newton sources.

\section{A.3. Optical Spectroscopy}

Since publishing the previous release of the Stripe $82 \mathrm{X}$ catalog, we have mined these additional databases for spectroscopic redshifts: PRIMUS (Coil et al. 2011), the Ross et al. (2012) pre-BOSS pilot survey with Hectospec, 6dF (Jones et al. 2004, 2009), and VVDS (Garilli et al. 2008). We also have an ongoing ground-based follow-up campaign to target X-ray sources and have redshifts from WIYN HYDRA from observing runs in 2012 December, 2013 August-September, 2014 January, June, July, September, and 2015 January; from ISAAC on VLT from 2013 August; from NIRSPEC on Keck 2013 September; and from Palomar DoubleSpec from 2014 July and December. We now have redshifts, and where available, optical classifications for 335 of the Chandra sources and 760 of the XMM-Newton sources; 142 of these redshifts are from our follow-up observing program, where the WIYN HYDRA spectra were reduced with the IRAF routine dohydra, the ISAAC spectrum was reduced with the VLT provided esorex pipeline, the NIRSPEC data were reduced with the IRAF task wmkonspec, and the Palomar DoubleSpec spectra were extracted using the conventional single-slit extraction routines in IRAF. Stars and extragalactic objects were classified on the basis of their emission and/or absorption features.

\section{A.4. AllWISE Catalog}

We now match the archival Chandra, archival XMMNewton, and AO10 XMM-Newton source lists to the AllWISE rather than AllSky Catalog, superseding the matches published in LaMassa et al. (2013a). We impose a $R_{\text {crit }}$ cut of 0.7 for the Chandra/AllWISE matching, finding five spurious associations out of 700. In total, 667 WISE sources survived the quality control cuts, where all 33 rejected sources were extended with either null or flagged extended photometry; two extended sources had acceptable elliptical aperture photometry measurements in at least one band. For the archival and AO10 XMM-Newton matching, our $R_{\text {crit }}$ value is 0.85 , with a spurious fraction of 20/1516. We were left with 1465 WISE sources that passed the quality control checks, of which four were extended. Forty-eight extended sources were rejected, as were three point sources. For reference, matching to the AllSky WISE catalog garnered 595 and 1398 Chandra and XMMNewton sources, respectively, so we increase the percentage of X-ray sources with WISE counterparts from $52 \%$ to $58 \%$ and $59 \%$ to $62 \%$, respectively. We note that the archival X-ray data have deeper pointings, causing the association rate to be lower than for the XMM-Newton AO13 source list which is at a relatively shallow depth.

\section{A.5. VHS}

Similar to the XMM-Newton AO13 catalog, we include columns for matching the archival Chandra, archival XMMNewton, and AO10 XMM-Newton source lists to the VHS catalog. We find critical $R_{\text {crit }}$ values of 0.85 for $J$ and 0.8 for $H$ and $K$ when matching to the Chandra source list, with an estimated 5/530,6/500, and 8/544 contamination rate in the $J$, $H$, and $K$ bands, respectively. In total, 577 VHS counterparts are found for the Chandra sources (50\% of the sample), with none rejected for being saturated. Between UKIDSS and VHS, there are NIR counterparts for 689 Chandra sources, or $60 \%$ of the source list. Of the $112 \mathrm{X}$-ray/UKIDSS objects without a VHS counterpart, 41 are undetected in the VHS survey; the remaining were below the $R_{\text {crit }}$ threshold.

When matching the archival and AO10 XMM-Newton catalog the VHS source list, we used a $R_{\text {crit }}$ threshold of 0.75 in the $J$ and $H$ bands and 0.8 in the $K$ band, with spurious association rates of $30 / 1250,27 / 1200$, and $38 / 1280$, respectively. We find 1504 VHS counterparts to the XMM-Newton sources (64\% of the sample), while 1670 X-ray objects $(71 \%)$ have NIR matches in either UKIDSS or VHS. Of the 166 X-ray/UKIDSS sources without a VHS match, 45 were undetected in the VHS survey.

\section{A.6. Herschel}

Several of the archival Chandra and XMM-Newton observations overlap the HerS survey area (Viero et al. 2014), so we use a nearest neighbor match to find counterparts to these X-ray sources. Again, the XMM-Newton search radius is $5^{\prime \prime}$. In LaMassa et al. (2013a), we used a $5^{\prime \prime}$ search radius to find counterparts to Chandra sources, though here we lower this search radius to $3^{\prime \prime}$ when matching to Herschel since our exercise of matching the randomized X-ray source positions to the Herschel catalog found false matches only at radii above $3^{\prime \prime}$. For both the Chandra and XMM-Newton matches to Herschel, no associations were found between the randomized X-ray positions and the Herschel source list.

\section{A.7. Catalog Cross-matches}

The previously released versions of the Stripe $82 \mathrm{X}$ multiwavelength matched catalogs did not include promoted matches found from cross-correlating the individual catalogs as discussed above. In the current versions of the catalogs, the promoted matches are included as well as flags to indicate which multi-wavelength counterparts are added into the catalog in this manner.

Additionally, we also have included a "cp_coord_flag," as described in the main text. The coordinates among the multiwavelength counterparts are consistent for $96 \%$ of the Chandra sources and for $92 \%$ of the XMM-Newton sources.

\section{REFERENCES}

Ahn, C. P., Alexandroff, R., Allende Prieto, C., et al. 2012, ApJS, 203, 21 Ahn, C. P., Alexandroff, R., Allende Prieto, C., et al. 2014, ApJS, 211, 17 Alam, S., Albareti, F. D., Allende Prieto, C., et al. 2015, arXiv:1501.00963 Alexander, D. M., Brandt, W. N., Hornschemeier, A. E., et al. 2001, AJ, 122, 2156

Annis, J., Soares-Santos, M., Strauss, M. A., et al. 2014, ApJ, 794, 120

Assef, R. J., Stern, D., Kochanek, C. S., et al. 2013, ApJ, 772, 26

Baldwin, J. A., Phillips, M. M., \& Terlevich, R. 1981, PASP, 93, 5

Becker, R. H., Helfand, D. J., White, R. L., Gregg, M. D., \& Laurent-Muehlheisen, S. A. 2012, yCat, 8090, 0

Becker, R. H., White, R. L., \& Helfand, D. J. 1995, ApJ, 450, 559

Bertin, E., \& Arnouts, S. 1996, A\&AS, 117, 393

Brandt, W. N., \& Alexander, D. M. 2015, A\&ARv, 23, 1

Brandt, W. N., \& Hasinger, G. 2005, ARA\&A, 43, 827

Brusa, M., Bongiorno, A., Cresci, G., et al. 2015, MNRAS, 446, 2394

Brusa, M., Civano, F., Comastri, A., et al. 2010, ApJ, 716, 348

Brusa, M., Zamorani, G., Comastri, A., et al. 2007, ApJS, 172, 353

Cappelluti, N., Brusa, M., Hasinger, G., et al. 2009, A\&A, 497, 635

Cappelluti, N., Hasinger, G., Brusa, M., et al. 2007, ApJS, 172, 341

Cardamone, C. N., Urry, C. M., Damen, M., et al. 2008, ApJ, 680, 130

Casali, M., Adamson, A., Alves de Oliveira, C., et al. 2007, A\&A, 467, 777

Civano, F., Marchesi, S., Comastri, A., et al. 2015, ApJ, in press 
Civano, F., Elvis, M., Brusa, M., et al. 2012, ApJS, 201, 30

Coil, A. L., Blanton, M. R., Burles, S. M., et al. 2011, ApJ, 741, 8

Croom, S. M., Richards, G. T., Shanks, T., et al. 2009, MNRAS, 392, 19 Delvecchio, I., Gruppioni, C., Pozzi, F., et al. 2014, MNRAS, 439, 2736

Donley, J. L., Koekemoer, A. M., Brusa, M., et al. 2012, ApJ, 748, 142

Drinkwater, M. J., Jurek, R. J., Blake, C., et al. 2010, MNRAS, 401, 1429

Efstathiou, A., \& Rowan-Robinson, M. 1995, MNRAS, 273, 649

Elvis, M., Civano, F., Vignali, C., et al. 2009, ApJS, 184, 158

Evans, I. N., Primini, F. A., Glotfelty, K. J., et al. 2010, ApJS, 189, 37

Farrah, D., Bernard-Salas, J., Spoon, H. W. W., et al. 2007, ApJ, 667, 149

Fowler, J. W., Niemack, M. D., Dicker, S. R., et al. 2007, ApOpt, 46, 3444

Frieman, J. A., Bassett, B., Becker, A., et al. 2008, AJ, 135, 338

Fritz, J., Franceschini, A., \& Hatziminaoglou, E. 2006, MNRAS, 366, 767

Garilli, B., Le Fèvre, O., Guzzo, L., et al. 2008, A\&A, 486, 683

Georgakakis, A., Aird, J., Buchner, J., et al. 2015, arXiv:1507.07558

Giacconi, R., Rosati, P., Tozzi, P., et al. 2001, ApJ, 551, 624

Glikman, E., Urrutia, T., Lacy, M., et al. 2013, ApJ, 778, 127

Green, P. J., Silverman, J. D., Cameron, R. A., et al. 2004, ApJS, 150, 43

Haardt, F., \& Maraschi, L. 1991, ApJL, 380, L51

Hasinger, G., Cappelluti, N., Brunner, H., et al. 2007, ApJS, 172, 29

Helfand, D. J., White, R. L., \& Becker, R. H. 2015, ApJ, 801, 26

Hewett, P. C., Warren, S. J., Leggett, S. K., \& Hodgkin, S. T. 2006, MNRAS, 367,454

Hodge, J. A., Becker, R. H., White, R. L., Richards, G. T., \& Zeimann, G. R. 2011, AJ, 142, 3

Hooper, E. J., Impey, C. D., Foltz, C. B., \& Hewett, P. C. 1995, ApJ, 445, 62 Hopkins, P. F., \& Hernquist, L. 2009, ApJ, 694, 599

Hsu, L.-T., Salvato, M., Nandra, K., et al. 2014, ApJ, 796, 60

Jiang, L., Fan, X., Bian, F., et al. 2014, ApJS, 213, 12

Jiang, L., Fan, X., Cool, R. J., et al. 2006, AJ, 131, 2788

Jones, D. H., Read, M. A., Saunders, W., et al. 2009, MNRAS, 399, 683

Jones, D. H., Saunders, W., Colless, M., et al. 2004, MNRAS, 355, 747

Kellermann, K. I., Sramek, R., Schmidt, M., Shaffer, D. B., \& Green, R. 1989, AJ, 98, 1195

Kenter, A., Murray, S. S., Forman, W. R., et al. 2005, ApJS, 161, 9

Kim, M., Kim, D.-W., Wilkes, B. J., et al. 2007, ApJS, 169, 401

Koratkar, A., \& Blaes, O. 1999, PASP, 111, 1

Lacy, M., Storrie-Lombardi, L. J., Sajina, A., et al. 2004, ApJS, 154, 166

Laird, E. S., Nandra, K., Georgakakis, A., et al. 2009, ApJS, 180, 102

LaMassa, S. M., Cales, S., Moran, E. C., et al. 2015a, ApJ, 800, 144

LaMassa, S. M., Civano, F., Brusa, M., et al. 2015b, arXiv:1511.02883

LaMassa, S. M., Urry, C. M., Cappelluti, N., et al. 2013a, MNRAS, 436, 3581

LaMassa, S. M., Urry, C. M., Glikman, E., et al. 2013b, MNRAS, 432, 1351

Lawrence, A., Warren, S. J., Almaini, O., et al. 2007, MNRAS, 379, 1599

Le Fèvre, O., Cassata, P., Cucciati, O., et al. 2013, A\&A, 559, A14

Le Fèvre, O., Saisse, M., Mancini, D., et al. 2003, Proc. SPIE, 4841, 1670

Le Fèvre, O., Vettolani, G., Garilli, B., et al. 2005, A\&A, 439, 845

Lehmer, B. D., Brandt, W. N., Alexander, D. M., et al. 2005, ApJS, 161, 21

Lehmer, B. D., Xue, Y. Q., Brandt, W. N., et al. 2012, ApJ, 752, 46

Loaring, N. S., Dwelly, T., Page, M. J., et al. 2005, MNRAS, 362, 1371
Luo, B., Brandt, W. N., Xue, Y. Q., et al. 2010, ApJS, 187, 560

Lutz, D., Maiolino, R., Spoon, H. W. W., \& Moorwood, A. F. M. 2004, A\&A, 418, 465

Magdis, G. E., Rigopoulou, D., Helou, G., et al. 2013, A\&A, 558, A136

Mainzer, A., Bauer, J., Grav, T., et al. 2011, ApJ, 731, 53

Maiolino, R., Comastri, A., Gilli, R., et al. 2003, MNRAS, 344, L59

Marchesi, S., Civano, F., Elvis, M., et al. 2015, arXiv:1512.01105

Mateos, S., Warwick, R. S., Carrera, F. J., et al. 2008, A\&A, 492, 51

McMahon, R. G., Banerji, M., Gonzalez, E., et al. 2013, Msngr, 154, 35

Meléndez, M., Heckman, T. M., Martínez-Paredes, M., Kraemer, S. B., \& Mendoza, C. 2014, MNRAS, 443, 1358

Merloni, A., Dwelly, T., Salvato, M., et al. 2015, MNRAS, 452, 69

Merloni, A., Predehl, P., Becker, W., et al. 2012, arXiv:1209.3114

Morrissey, P., Conrow, T., Barlow, T. A., et al. 2007, ApJS, 173, 682

Mullaney, J. R., Alexander, D. M., Goulding, A. D., \& Hickox, R. C. 2011, MNRAS, 414, 1082

Murray, S. S., Kenter, A., Forman, W. R., et al. 2005, ApJS, 161, 1

Netzer, H., Lutz, D., Schweitzer, M., et al. 2007, ApJ, 666, 806

Newman, J. A., Cooper, M. C., Davis, M., et al. 2013, ApJS, 208, 5

Pier, E. A., \& Krolik, J. H. 1992, ApJ, 401, 99

Predehl, P., Andritschke, R., Becker, W., et al. 2014, Proc. SPIE, 9144, 91441 T

Ranalli, P., Comastri, A., Vignali, C., et al. 2013, A\&A, 555, A42

Ranalli, P., Georgantopoulos, I., Corral, A., et al. 2015, A\&A, 577, A121

Rosario, D. J., Santini, P., Lutz, D., et al. 2012, A\&A, 545, A45

Rosen, S. R., Webb, N. A., Watson, M. G., et al. 2015, arXiv:1504.07051

Ross, N. P., Myers, A. D., Sheldon, E. S., et al. 2012, ApJS, 199, 3

Rots, A. H., \& Budavári, T. 2011, ApJS, 192, 8

Schartmann, M., Meisenheimer, K., Camenzind, M., et al. 2008, A\&A, 482, 67

Schmidt, M., Hasinger, G., Gunn, J., et al. 1998, A\&A, 329, 495

Schweitzer, M., Lutz, D., Sturm, E., et al. 2006, ApJ, 649, 79

Shao, L., Lutz, D., Nordon, R., et al. 2010, A\&A, 518, L26

Spinoglio, L., \& Malkan, M. A. 1989, ApJ, 342, 83

Stern, D., Assef, R. J., Benford, D. J., et al. 2012, ApJ, 753, 30

Stern, D., Eisenhardt, P., Gorjian, V., et al. 2005, ApJ, 631, 163

Sutherland, W., \& Saunders, W. 1992, MNRAS, 259, 413

Swetz, D. S., Ade, P. A. R., Amiri, M., et al. 2011, ApJS, 194, 41

Treister, E., Schawinski, K., Urry, C. M., \& Simmons, B. D. 2012, ApJL, 758, L39

Vanden Berk, D. E., Richards, G. T., Bauer, A., et al. 2001, AJ, 122, 549

Veilleux, S., \& Osterbrock, D. E. 1987, ApJS, 63, 295

Viero, M. P., Asboth, V., Roseboom, I. G., et al. 2014, ApJS, 210, 22

Warren, S. J., Hambly, N. C., Dye, S., et al. 2007, MNRAS, 375, 213

Watson, M. G., Schröder, A. C., Fyfe, D., et al. 2009, A\&A, 493, 339

Weaver, K. A., Meléndez, M., Mushotzky, R. F., et al. 2010, ApJ, 716, 1151

White, R. L., Becker, R. H., Helfand, D. J., \& Gregg, M. D. 1997, ApJ, 475,479

Wright, E. L., Eisenhardt, P. R. M., Mainzer, A. K., et al. 2010, AJ, 140, 1868

Xue, Y. Q., Luo, B., Brandt, W. N., et al. 2011, ApJS, 195, 10

York, D. G., Adelman, J., Anderson, J. E., Jr., et al. 2000, AJ, 120, 1579 